Trabajo seleccionado para su publicación por el jurado del Premio Estudios Financieros, formado por: don José Ignacio Baile Ayensa, don Julio Cabero Almenara, doña Trinidad Manzano Moreno, don Antonio Pastor Sanmillán, don Juan Pazos Sierra y don Ismael Sanz Labrador.

\title{
Nuevos entornos abiertos de aprendizaje para la enseñanza de lenguas extranjeras. ¿Son capaces los MOOC de generar un cambio metodológjco?
}

\section{Extracto:}

\section{Sumario}

1. Introducción

2. Objetivos

3. Marco teórico

4. Iversity

5. MOOC para la enseñanza del español como lengua extranjera

6. Resultados

7. Discusión

8. Conclusiones y retos

9. Bibliografía

Apéndices

El nuevo contexto en el que se enmarca la educación viene determinado por la sociedad-red que está surgiendo, ya que la implementación de nuevos enfoques dirigidos hacia la formación y el conocimiento se realizan a través de internet, como medio para la enseñanza y el aprendizaje. Diseñar los contenidos educativos y la estructura de un curso para el aprendizaje desde la enseñanza online ha conducido a explorar nuevos modelos e-learning, especialmente en la enseñanza universitaria. El gran interés originado por los massive open online courses (MOOC) ha dado lugar al desarrollo y perfeccionamiento de las plataformas en abierto, presentando un escenario formativo en el que definir un método de enseñanza-aprendizaje acorde con la actualidad del momento y con las necesidades de la sociedad, que sea capaz de resolver el binomio tecnología-pedagogía. Se hace necesario analizar la estructura y el diseño de estos cursos masivos, en abierto y en línea, y definir sus características curriculares, es decir, aquellas que tienen que ver con la forma de entender la enseñanza y el aprendizaje. En este trabajo se propone el análisis, el diseño y la implementación de un MOOC para el aprendizaje del español como lengua extranjera con el objetivo de explorar las posibilidades educativas de los nuevos entornos de aprendizaje para la formación y el conocimiento de lenguas, e indagar sobre si son capaces de generar un cambio metodológico. Este trabajo pretende abrir nuevas vías de investigación y reconocer los nuevos avances tecnológicos para la enseñanza de lenguas.

\footnotetext{
J. Bravo-Agapito, profesor doctor del Departamento de Ingeniería Informática y de Telecomunicaciones de la Universidad a Distancia de Madrid (UDIMA).

${ }^{2}$ A. Centellas Rodrigo, directora del Instituto de Idiomas de la Universidad a Distancia de Madrid (UDIMA).

${ }^{3}$ R. Aguayo Sarasa, profesora del Máster en Educación y Nuevas Tecnologías.
} 


\section{New open learning environments}

\section{for second language} acquisition. Are the

MOOCs able to boost a methodological change?

\section{Abstract:}

The new context in which education is framed is determined by the network society that is emerging as the implementation of new approaches directed towards training and knowledge are carried out through the internet, as a means for teaching and learning. Designing the educational contents and the structure of a course for learning from online teaching has led to explore new e-learning models, especially in university teaching. The great interest originated by the massive open online courses (MOOCs) has given rise to the development and improvement of the open platforms, presenting a formative scenario in which to define a teaching-learning method according to the current situation and with the needs of the society, capable of to solve the binomial technology-pedagogy. It is necessary to analyze the structure and design of these massive courses, open and online and define their curricular characteristics, those that have to do with the way of understanding teaching and learning. This paper proposes the analysis, design and implementation of a MOOC for the learning of Spanish as a foreign language with the aim of exploring the educational possibilities of new learning environments for language training and knowledge and to investigate if they are capable to generate a methodological change. This work aims to open new avenues of research and recognize new technological advances for the teaching of languages.

Keywords: massive open online courses (MOOCs), language learning, Spanish, Iversity.

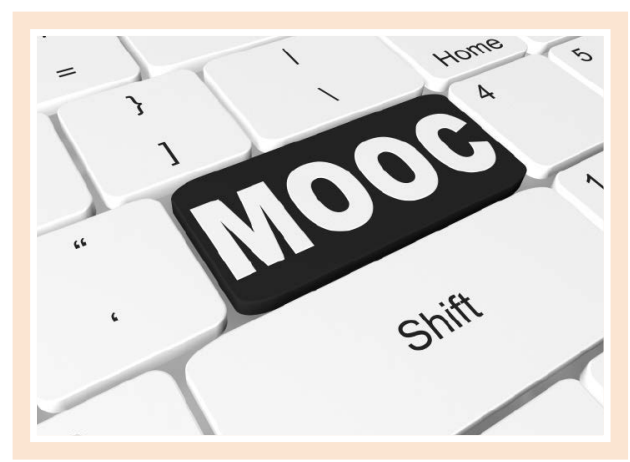

\section{INTRODUCCIÓN}

En la última década, la educación universitaria ha evolucionado de forma significativa debido a la amplia adopción de los sistemas e-learning por las universidades y centros educativos. Por un lado, la tecnología de la información y las comunicaciones (TIC) ha facilitado esta adopción, pues proporciona nuevas formas de transmitir, organizar y presentar los contenidos educativos. Por ejemplo, profesores y estudiantes pueden utilizar nuevos dispositivos, como smartphones, tablet $P C$, lectores digitales electrónicos (eReaders), etc., pero también tienen a su disposición nuevos protocolos de comunicación como SMS (short message service), Bluetooth, wifi, NFC (near field communication), etc., y también nuevas aplicaciones y sistemas informáticos como learning management systems (LMS) y laboratorios virtuales, entre otros, que facilitan y flexibilizan el proceso enseñanza-aprendizaje (Chen, Myers y Yaron, 2000). Por otro lado, el Espacio Europeo de Educación Superior (EEES) ha promocionado y fomentado la utilización de estas nuevas tecnologías con el objetivo de mejorar el proceso de aprendizaje de los estudiantes europeos.

Debido a que los estudiantes pueden tener diferentes características (por ejemplo, distintas metas, intereses, conocimientos previos, culturas o estilos de aprendizajes), sería deseable adaptar el proceso de aprendizaje de cada estudiante teniendo en cuenta estas características. Este es el principal objetivo de los sistemas adaptativos hipermedia orientados a la enseñanza (Brusilovsky, 1996), que son una evolución de los sistemas e-learning. Estos sistemas son capaces de adaptar tanto los contenidos educativos como 
la navegación entre estos con el objetivo de guiar de forma automática a los estudiantes de acuerdo a sus características personales. Ejemplos de su éxito son los conocidos sistemas task-based adaptive learner guidance on the web (TANGOW) (Carro, Pulido y Rodríguez, 1999), QuizGuide (Sosnovsky y Brusilovsky, 2005) y cognitive tutor authoring tools (CTAT) (Aleven, McLaren, Sewall y Koedinger, 2006).

Mientras que esta corriente se concentró en mejorar la experiencia de enseñanza-aprendizaje, enfocándose principalmente en el proceso de aprendizaje, en el año 2008 surgió una nueva corriente cuyo objetivo era transmitir contenidos educativos a través de un curso online de forma abierta a un gran número de estudiantes. A estos cursos se los denominó con el término $\mathrm{MOOC}$, es decir, cursos a través de internet con un número masivo de estudiantes y de acceso libre (Salvi y Bravo, 2013).

Según Daniel (2012), los MOOC se pueden clasificar en dos tipos: CMOOC y xMOOC. Los primeros se basan en la teoría conectivista, que establece que el conocimiento está distribuido en una red de conexiones y que el aprendizaje consiste en la habilidad de asociar estas conexiones para formar estas redes (Downes, 2007). Los segundos siguen el modelo de Silicon Valley, o también conocido como modelo Coursera, y se enfocan fundamentalmente en un modelo de enseñanza conductivista en el que el aprendizaje se genera a través de vídeos y actividades de autoevaluación.

Un hecho destacable es que la evolución de estos cursos ha sido exponencial durante el año 2012, sobre todo de los XMOOC. Por ejemplo, en Europa, en ese año, se crearon $28 \mathrm{MOOC}$, y en el año 2016, más de 1.700 , lo que supone un incremento de más del $6.000 \%$ en cuatro años, pero se estima que a principios del año 2018 se llegue a más de 1.800 (Open Education Europa, 2016).

El aprendizaje de una lengua distinta a la materna ha sido objeto de estudio desde la antigüedad. Un ejemplo de esto lo encontramos en el siglo III d. C. en el Hermeneumata, libro que se utilizaba para que los hablantes en latín aprendieran también griego. Este libro estaba escrito por un lado en latín y por el otro en griego, por lo que los estudiantes podían aprender griego comparando las oraciones y el vocabulario; sin embargo, este libro no incluía explicaciones sobre las estructuras gramaticales. Siguiendo esta línea, es en el siglo XX cuando se establece un debate sobre el aprendizaje huma- no entre la corriente basada en el comportamiento y la basada en la psicología cognitiva. Este debate tuvo su punto central en cómo debía ser la adquisición de una segunda lengua (second language acquisition [SLA]), que se estableció como una nueva línea de investigación (Larsen-Freeman y Long, 1991). Se puede decir que una evolución de la SLA es la enseñanza de idiomas asistida por ordenador (computer assisted language learning [CALL]), campo de investigación que une la enseñanza de idiomas con la ingeniería informática. Así, el presente trabajo se encuentra enmarcado en el campo de investigación de la CALL.

En este trabajo se propone el análisis, el diseño y la implementación de un MOOC para el aprendizaje del español como lengua extranjera. Es importante destacar que el MOOC diseñado e implementado es del tipo XMOOC y fue desarrollado de forma completa por el equipo de investigación desde su fase embrionaria hasta la fase final, donde se realizó su primer lanzamiento a través de la plataforma Iversity el 22 de abril de 2015.

\section{OBJETIVOS}

En este trabajo se plantearon los siguientes objetivos:

- Explorar las posibilidades educativas de los nuevos entornos de aprendizaje para la formación y el conocimiento de lenguas.

- Analizar y valorar el diseño metodológico empleado en la creación de un curso de aprendizaje del español en el entorno del MOOC.

- Exponer las consecuencias que supone la implementación de un MOOC para la enseñanza de una lengua extranjera.

- Abrir nuevas vías de investigación y reconocer nuevos avances tecnológicos para la enseñanza de lenguas.

\section{MARCO TEÓRICO}

La revolución educativa generada por el desarrollo de las tecnologías está fomentando que el sujeto sea cada vez más crítico, más creativo, más autónomo y más consciente de su aprendizaje. En el contexto actual, hablar de educación implica enmarcarla dentro de 
una sociedad en la que la TIC es el nuevo escenario para la formación. Ya no se trata de que el aprendizaje "ocurra». Se trata de que «suceda» desarrollando nuevas teorías, enfoques, pedagogías, modalidades de aprendizaje, tecnologías, etc., que se guiarán por criterios diferentes en función de la visión de la realidad social del momento, según la época en la que nos encontremos. Cada sociedad adjudica un significado a los nuevos medios desde el contexto que los rodea. "Todas las culturas y edades tienen un modelo preferido de percepción y conocimiento que suelen prescribir para todo y a todos» (McLuhan, 1996, pág. 27). La introducción de nuevos medios en la sociedad de cada época ha supuesto cambios sustanciales en el desarrollo de la cultura, en la comunicación, y nuevos procesos de aprendizaje. ¿Cómo podemos hacer para que nuestros alumnos sean un público interactivo en este contexto específico de aprendizaje? ¿Qué ocurre cuando ese proceso de enseñanza se da a través de los medios de comunicación? ¿Qué sucede cuando escuchamos, leemos, hablamos, escribimos y aprendemos a través de los medios?

Hay que ver el aprendizaje como un producto de la autoorganización educacional. La aventura de diseñar el aprendizaje desde la enseñanza online nos lleva a explorar nuevos modelos e-learning, aprovechando el asombro como estímulo y la capacidad para trabajar dentro de estos entornos de aprendizaje. Internet ha conseguido crear equipo de trabajo entre el docente y el alumno, cada uno desempeñando sus funciones. El primero pone en marcha el proceso, satisface su curiosidad y plantea preguntas que provocan la reflexión y que requieren el trabajo en equipo del segundo. No obstante, no todo son ventajas, ya que internet presenta riesgos que hay que conocer para evitarlos. Uno de ellos es confundir datos, elementos inconexos, con el saber, que es siempre construcción integradora. Otro, reducir nuestras preguntas a aquellas para las que el computador pueda tener contestación (Landeta, 2012, págs. 21-25). La clave está en asombrarse del aprendizaje y plantear una pregunta que signifique un paso adelante hacia el conocimiento.

Debemos pensar en el e-learning como un modelo de aprendizaje intelectual donde la formación y el saber se generan de forma cooperativa y diagonal, con el apoyo de las herramientas y los recursos que nos ofrece la TIC.

\subsection{La enseñanza de lenguas y las tecno- logías: marco situacional}

Como se ha mencionado anteriormente, internet, desde los años sesenta, ha supuesto una gran revolución.

Somos testigos de la enorme revolución en la que estamos inmersos. Protagonistas de los avances tecnológicos, vamos controlándolo «todo» (por ejemplo, relaciones, contactos, cuentas bancarias, billetes de avión, etc.). El cambio tecnológico nos resulta tan cotidiano, tan cercano, que, en la mayoría de los casos, no tenemos conciencia de lo que está sucediendo. Cuando hablamos de nuevas tecnologías nos referimos a subtecnologías que están incluidas en la TIC, porque facilitan que la información y la comunicación fluyan entre la sociedad. Por otro lado, el nacimiento de una sociedad de la información como consecuencia de la revolución de las nuevas tecnologías no debe eclipsar los dos principios básicos que priman en cualquier sociedad: el conocimiento y la comunicación (Hafez, 2011; Thommen, 2008). La información es efectivamente un instrumento del conocimiento, pero no es el conocimiento en sí. Este, a lo largo de la historia, ha sido el motor de desarrollo y, por tanto, de avance y aparición de las nuevas sociedades. El conocimiento no se adquiere por estar informado, sino que debemos ser capaces de organizar y de interiorizar esa información. Alcanzamos conocimientos cuando ordenamos, articulamos y le damos sentido a una información procedente de los medios en un contexto determinado. En este sentido, la sociedad del conocimiento no es algo que haya surgido de repente, es más bien una etapa evolutiva hacia la que poco a poco ha ido avanzando la humanidad. Este fenómeno sociológico que ha ido creciendo exponencialmente ha constituido la universalidad de un acceso y un lenguaje que nos permite leer, escribir y participar, haciendo posible el aprendizaje online.

En las aulas siempre se han utilizado materiales reales didácticos que, sin ser digitales o tecnológicos, facilitaban la comunicación y el intercambio de información (por ejemplo, periódicos, revistas, folletos informativos, cintas de vídeo y audio, etc.). Hoy día las tecnologías están presentes, convergen, y es importante su incorporación en la enseñanza de las lenguas extranjeras, pues ayudan a aumentar el grado de conocimiento, asi como las habilidades de los discentes. Jenkins (2009) 
habla de la importancia de que los jóvenes adquieran la competencia digital y aquellas habilidades del siglo $\mathrm{XXI}$ que les permitan interactuar con la información y la cultura de hoy para convertirse en artistas creativos, en ciudadanos, en futuros trabajadores. La TIC, por tanto, conecta al alumno con el mundo exterior, y esto hace que el docente no se quede al margen. Al igual que estamos al día en avances psicopedagógicos, metodológicos y didácticos, también debemos estarlo en torno a las herramientas y a los recursos que nos permitan comunicar mejor esos conocimientos.

\subsection{Teorías de aprendizaje, TIC y ense- ñanza de lenguas extranjeras}

La integración de la TIC al aula, en general, y a la enseñanza de segundas lenguas (L2), en particular, implica el reajuste de los modelos de enseñanza a unas condiciones de aprendizaje que promueven la autonomía y el enfoque centrado en el alumno (Pérez y Pérez, 2006). La TIC fomenta un aprendizaje constructivo y significativo del lenguaje, es decir, el alumno es el que construye conocimiento al integrar lo nuevo a los conocimientos que tiene, que conoce. El proceso es controlado por los estudiantes, y sus resultados van a depender de ellos. Desde esta perspectiva el docente no puede transferir conocimientos, debe fomentar las habilidades necesarias para que cada alumno construya conocimiento de forma autónoma. De esta manera el aprendizaje será más significativo y motivador, creando entornos de trabajo más cooperativos, aumentando su independencia y convirtiendo al docente en facilitador del proceso.

Esta propuesta teórica, de enseñanza de una lengua extrajera, relaciona el uso de la TIC en el aula con la teoría del aprendizaje constructivista, o dicho de otra manera, el uso de la TIC en el aula favorece el aprendizaje constructivo, que huye del aprendizaje tradicional, basado en la instrucción.

La incorporación de la tecnología y sus formas de interacción rompe los monopolios tradicionales del sistema educativo y provoca un rediseño radical en las metodologías de aprendizaje, motivado por el propio contexto de la sociedad-red que está surgiendo. Como dicen Roig y Fernández (2015), si se educa en medios, se puede fomentar la creación de individuos críticos, creativos, conscientes de la realidad, capaces de actuar y aprender libre, autónoma y juiciosa- mente. No obstante, las tecnologías por sí mismas no garantizan el aprendizaje, sino que aportan los recursos que lo facilitan dentro de una programación educativa. Teniendo en cuenta esto, es necesario hablar de algunas de las razones por las cuales la TIC puede ayudar en el proceso de enseñanza-aprendizaje de una lengua extranjera: por un lado, el material real, es decir, la muestra de lengua que ofrecen los materiales digitales, y por otro, el papel que las herramientas y recursos tecnológicos desempeñan en el proceso de aprendizaje, haciendo que el alumno se convierta en receptor activo, en el centro del proceso, en creador de conocimiento. La participación es indispensable para aprender en entornos virtuales, aunque convergen otros factores que influyen muy positivamente en dicho proceso: la motivación, la actitud, el acceso y la autodisciplina. Por otra parte, no hay que olvidar, desde el punto de vista didáctico, que el carácter innovador del entorno de aprendizaje no implica que el aprendizaje sea óptimo y/o que se garantice su éxito.

Las posibilidades de aprendizaje que ofrece la TIC permiten escenarios formativos con nuevas propuestas metodológicas de enseñanza-aprendizaje, entre los que se pueden destacar los MOOC.

\subsection{Las plataformas e-learning como he- rramientas para la enseñanza de len- guas extranjeras}

Las plataformas e-learning, según Fernández-Pampillón (2009, págs. 45-47), «constituyen, actualmente, una realidad tecnológica creada en internet que da soporte a la enseñanza-aprendizaje universitaria». Este autor afirma que su uso ha transformado una gran parte de los espacios de enseñanza tradicionales en espacios virtuales de enseñanza y aprendizaje. Un claro ejemplo de esta afirmación son los MOOC.

Los MOOC proponen un nuevo modelo de enseñanza accesible desde cualquier ubicación y en cualquier instante de tiempo. Una característica que los distingue de otros sistemas e-learning es que existen una serie de plataformas que alojan los cursos, de forma que estas plataformas mantienen informados a sus usuarios de los nuevos cursos que van ofreciendo. Estas plataformas proporcionan distintas herramientas a los creadores de un MOOC; por ejemplo, un editor de cursos para añadir nuevas actividades, adaptadas a la gran cantidad de usuarios. 
Actualmente, existen multitud de plataformas, pero podemos seleccionar las siguientes como las desarrolladas con más éxito: edX, Udacity, Coursera, FutureLearn, MiriadaX e Iversity. La plataforma Iversity se explicará con más detalle en el epígrafe 4 .

$E d X^{4}$ es una plataforma creada por el Massachusetts Institute of Technology y la Harvard University con una dotación inicial de 60 millones de dólares. Esta plataforma ofrece actualmente 1.895 cursos, de los cuales 1.628 se ofertan en inglés y solo 141 están en lengua española. Esta plataforma sigue el modelo XMOOC, pero actualmente están enriqueciendo los cursos con la metodología de los cMOOC.

Udacity 5 fue creada por Sebastian Thrun y Peter Norvig en 2012 con una inversión inicial de 21,1 millones de dólares. Esta iniciativa nació como un experimento de la Stanford University. Esta plataforma ofrece fundamentalmente cursos en inglés de materias vinculadas con las ciencias y especialmente con la tecnología. Por ejemplo, actualmente oferta 28 cursos relacionados con Android ${ }^{6}$. La plataforma cuenta con dos tipos de cursos, los de acceso libre o los que están relacionados con el desarrollo de proyectos, llamados nanodegrees.

Coursera ${ }^{7}$ fue creada por Andrew $\mathrm{Ng}$ y Daphne Koller, de la Stanford University, con una inversión inicial de 22 millones de dólares. Coursera contiene cursos de distintas materias: ciencias, arte y humanidades. Respecto a los cursos de idiomas, esta plataforma ofrece 13 cursos para aprender idiomas, de los cuales 5 cursos son para aprender español. Coursera colabora con 149 instituciones (entre las que destacan International Business Machines [IBM], Sapienza-Università di Roma, University of London, University of Pittsburgh, Duke University, Yale University y Stanford University), distribuidas en 29 países. Actualmente esta plataforma ofrece más de 2.000 cursos.

FutureLearn ${ }^{8}$ es una iniciativa de la Open University que lanzó sus primeros cursos en el último trimestre

\footnotetext{
4 URL de edX: https://www.edx.org.

5 URL de Udacity: https://www.udacity.com.

6 Android es el sistema operativo desarrollado por Google para los dispositivos móviles.

7 URL de Coursera: https://www.coursera.org/.

8 URL de FutureLearn: https://www.futurelearn.com
}

del año 2013. La plataforma ofrece cursos de distintas materias, incluidas historia y lenguas y culturas. En el caso de cursos de idiomas, esta plataforma cuenta con 27 cursos en inglés para aprender idiomas, pero no oferta ninguno en español, ni para aprender español.

MiriadaX ${ }^{9}$ es una plataforma española que surge de la iniciativa de Universia y Telefónica Educación Digital y ofrece cursos fundamentalmente en español. De hecho, está presente en más de 1.300 universidades iberoamericanas. Los cursos que oferta son de diversas temáticas. MiriadaX oferta actualmente 690 cursos y colabora con 105 universidades, entre las que se encuentra la UDIMA. Respecto al aprendizaje de segundas lenguas, esta plataforma cuenta con 11 cursos, entre los cuales hay 2 para el aprendizaje del español: Instrucción Gramatical y Virtual ELE (Universidad de La Laguna) y Español Salamanca A2 (Universidad de Salamanca).

\subsection{MOOC}

El gran interés de los $\mathrm{MOOC}$ ha originado el desarrollo y perfeccionamiento de plataformas para la formación en abierto. Los MOOC nos presentan un nuevo escenario formativo en el que definir un método de enseñanza-aprendizaje acorde al contexto actual, ya que es la plataforma la que va a determinar el diseño metodológico, dato importante si no queremos caer en la creación de un MOOC para enseñar una lengua extranjera con énfasis en un aprendizaje tradicional, al centrarse, por ejemplo, en la visualización de vídeos y la realización de ejercicios tipo test.

Investigadores como Regalado (2012) opinan que los MOOC son la innovación tecnológica en educación de las últimas décadas, pudiendo producir cambios significativos en el sistema educativo. Por lo tanto, se hace necesario profundizar en la estructura y en el diseño metodológico de los mismos a la hora de ofertar cursos masivos, abiertos y en línea, ya que estos van a definir desde la política educativa de la universidad hasta sus características curriculares, es decir, aquellas que tienen que ver con la forma de entender la enseñanza-aprendizaje.

9 URL de MiriadaX: https://miriadax.net. 
El MOOC que se presenta sigue una metodología ecléctica. Eso quiere decir que la educación tradicional no va a desaparecer, pero sí se va a trasformar, al intentar combinar la tecnología con las actuales líneas metodológicas; en otras palabras, ajustar los contenidos a las necesidades interactivas de los estudiantes.

\section{IVERSITY}

Se trata de una plataforma europea virtual para el alojamiento y la realización de cursos MOOC, orientados a la educación superior y a la formación empresarial. De origen alemán, cuenta con más de 700.000 usuarios en todo el mundo, según Chang (2014), y está disponible en inglés y alemán, aunque algunos cursos pueden encontrarse en otros idiomas, como ruso, italiano y español.

Considerada como la plataforma europea de cursos masivos en abierto por excelencia, Iversity cuenta con un gran número de usuarios, de los cuales, una inmensa mayoría son europeos, pero sus acciones formativas son seguidas por personas de distintas y variadas nacionalidades a nivel mundial, como, por ejemplo, Filipinas, EE. UU., Japón, India, etc.

En esta plataforma, las temáticas de los cursos son muy diversas, pudiendo encontrar desde cursos de matemáticas, ciencias o tecnología, hasta cursos de humanidades, aunque destacan ligeramente aquellas acciones formativas basadas en temática de negocios $y$ empresas.

Los cursos que ofrece Iversity son diseñados, proporcionados y realizados por distintas universidades europeas, entidades y empresas de gran relevancia. Algunas de las universidades colaboradoras que participan con la plataforma en la realización de cursos son la University of Buckingham, la UDIMA, la University of Applied Sciences Berlin, la Università degli Studi di Pavia, etc., y entre las distintas instituciones y empresas que colaboran con Iversity en la creación de cursos para el fomento del desarrollo profesional se encuentran la Comisión Europea, el European University Institute, etc.

Introducir la empresa como un elemento formador ha supuesto para esta plataforma alemana la posibilidad no solo de ofrecer cursos orientados a la formación en la educación superior, sino también poder ofertar pa- quetes de formación para empresas en cuestiones de comunicación empresarial y transformación digital de la empresa, dos temáticas muy demandadas actualmente en el sector empresarial.

Hay que destacar que Iversity ofrece a las universidades y centros educativos la posibilidad de crear cursos con valoración en créditos ECTS (european credit transfer and accumulation system) para que sean fácilmente convalidables dentro del EEES.

Superar un curso en esta plataforma supone obtener un certificado, que puede ser de dos tipos:

- Certificado de participación. Se obtiene de manera gratuita, habiendo superado un porcentaje del curso, previamente establecido por el equipo docente. Este porcentaje suele estar por encima del $80 \%$.

- Certificado de superación. Se obtiene pagando una cantidad determinada, después de haber superado un porcentaje del curso, previamente establecido por el equipo docente. Al igual que en el caso anterior, este porcentaje suele ser superior al $80 \%$.

Esta plataforma cuenta con un sistema de identificación facial del estudiante, conocido como online proctoring, que permite identificar a la persona que realiza el examen final, con la intención de evitar que sean otras personas las que le suplanten en el examen.

\subsection{Entorno}

Iversity cuenta con un entorno amigable y muy intuitivo, que permite al usuario realizar los distintos cursos que se ofrecen de una manera sencilla y atractiva.

Los recursos didácticos y estructurales que ofrece esta plataforma para la realización y el montaje de cursos en este formato son diversos. A continuación, se presentan algunos de los recursos didácticos que facilitan y mejoran la adquisición y comprensión de contenidos por parte de los usuarios/alumnos en los cursos:

- Vídeo. La plataforma está preparada para el alojamiento de material en formato audiovisual a través de tres métodos de subida distintos: URL ${ }^{10}$

10 En este trabajo trataremos las direcciones URL como la dirección lógica de una máquina en internet. Así, entenderemos como direcciones válidas aquellas precedidas por el protocolo HTTP o HTTPS. Por ejemplo, https://iversity.org/ es una dirección URL válida. 
(uniform resource locator), archivo local y archivo en Dropbox ${ }^{11}$. Iversity recomienda vídeos con un máximo de 10 minutos de duración, ya que después de este tiempo hay un riesgo de pérdida de atención de los estudiantes. A continuación se describen los tres métodos:

- URL. Este método de subida de material audiovisual permite el alojamiento de vídeos en la plataforma a través de enlaces extraídos de plataformas como YouTube, Vimeo, etc.

- Archivo local. Permite subir vídeos alojados previamente en un equipo, siempre que no sobrepase las limitaciones de peso que impone la plataforma.

- Archivo en Dropbox. La plataforma da la posibilidad de incluir en los cursos vídeos que se tengan previamente alojados a través del servicio Dropbox.

- Texto. Puede incluirse en el curso incrustado en la plataforma o subido en formato archivo de texto. Para el texto incrustado, Iversity cuenta con editor de texto simple que permite realizar textos acompañados de imágenes y direcciones web; en cambio, para el alojamiento de textos en formato PDF (portable document format), la plataforma cuenta con las mismas opciones de subida que en los vídeos, es decir, subida de los textos a la plataforma a través de una URL mediante archivos locales 0 a través del servicio Dropbox.

- Imágenes. Iversity permite la introducción de imágenes dentro del curso en diversos apartados, como, por ejemplo, en los materiales complementarios, acompañando a los textos, e incluso como complemento en las actividades didácticas. Las opciones de subida de imágenes a la plataforma son las anteriormente mencionadas en los apartados de vídeos y textos, teniendo una limitación en el peso de los documentos visuales en $50 \mathrm{MB}^{12}$.

- Actividades didácticas. Las actividades que proporciona Iversity son diversas en cuanto a tipología y se clasifican en tres tipos:

\footnotetext{
11 Dropbox es un servicio para alojar archivos en un espacio en internet que se conoce como «nube». Una cuenta estándar en este servicio proporciona al usuario un espacio de 5 GB (aproximadamente 5 millones de bytes)

$121 \mathrm{MB}$ equivale a 1.024 bytes. El byte es la unidad básica de información de un computador y equivale a 8 bits.
}

- Autocorregibles. Este tipo de actividades permiten a los estudiantes ver las respuestas inmediatamente después de terminarlas (short test) 0 después de un tiempo determinado por el equipo docente (homework). En el caso de las actividades de tipo short test, el cuestionario se compone de 3 a 4 preguntas. Para estas actividades, el feedback que proporciona la plataforma es amigable, ya que, en el caso de acierto, mostrará una cara sonriente de color verde y, en el caso de fallo, una cara de color rojo. Además, estas actividades se integran de forma adecuada en la plataforma, pudiendo ubicar esta actividad en la misma ventana que el vídeo. De esta forma, los estudiantes pueden avanzar, retrasar, pausar el vídeo mientras responden las preguntas, lo cual es muy útil para el aprendizaje de idiomas, ya que este tipo de aprendizaje requiere práctica y repetición de estructuras. Las actividades de tipo homework se utilizan para evaluar el progreso del estudiante en el curso. Estas actividades se componen de una serie de preguntas, que serán evaluadas de forma automática después de una fecha fijada por el equipo docente. Respecto a los tipos de preguntas que se pueden introducir en cualquiera de estas actividades, la plataforma nos ofrece tres clases:

- «Preguntas de respuesta simple». En este tipo de preguntas, solo una respuesta es la correcta.

- «Preguntas de respuesta múltiple». En este tipo de preguntas, varias respuestas son correctas.

- «Preguntas de respuesta corta». En este tipo de preguntas, los usuarios deben responder a través de un editor de texto que les permite contestar de manera breve y concisa. Además, estas preguntas se pueden evaluar a través de expresiones regulares, indicadas por el equipo docente.

Otra característica relevante que ofrece Iversity sobre estas actividades es que pueden ser parametrizadas de diversas formas, para adaptar la actividad a sus necesidades didácticas; por ejemplo, adecuar la puntuación de cada una de las preguntas, incluir imágenes, introducir URL que sean necesarias para la realización del ejercicio.

- P2P. Estas actividades son corregidas por los mismos usuarios, entre ellos, permitiendo que los correctores puedan realizar comentarios 0 feedbacks al usuario que está corrigiendo. 
- Exámenes. El sistema cuenta con un recurso para realizar exámenes parciales y finales, basado en preguntas de tipo autocorregible, en el que el usuario pueda demostrar los conocimientos adquiridos durante el curso. Estos exámenes, sobre todo los finales, son primordiales para la consecución de la certificación.

Además de recursos didácticos que enriquecen los cursos, Iversity cuenta con una herramienta de comunicación asincrónica, en este caso un "foro de discusión», donde los usuarios pueden realizar sus preguntas al equipo docente encargado del curso para aclarar dudas respecto al contenido, comunicarse entre ellos y realizar, a través de sus aportaciones, una construcción colaborativa y colectiva del conocimiento. Hay que destacar que, en este foro, los estudiantes pueden seleccionar la unidad objeto de la duda. Además, el foro de discusión permite establecer un ranking de mensajes, teniendo en cuenta las votaciones de los estudiantes en cada entrada del foro. Esta característica es muy útil para el equipo docente, ya que permite que este responda a las preguntas más votadas, que son las más relevantes para los estudiantes.

\subsection{Estructura de un curso}

Los cursos dentro de la plataforma Iversity suelen tener una estructura basada en bloques temáticos 0 temas $^{13}$ (Iversity, 2015). El orden de los elementos dentro de los bloques temáticos, y de estos mismos, no está regido por ningún patrón previamente impuesto, sino que cada institución diseña el curso de la manera que le parece más conveniente, pudiendo introducir una cantidad de recursos de acuerdo al diseño previamente planificado. Hay que señalar que el equipo docente está en contacto con un equipo de Iversity, designado para cada MOOC, que consensuará el diseño o la estructura final del curso.

Cada bloque temático o tema se divide en unidades de trabajo que contienen un elemento o recurso didáctico (vídeo, texto o imagen), el cual se utiliza para ofrecer la información teórica, y pueden ser completadas con actividades didácticas autocorregibles, y material complementario en formato texto, imagen o URL, para la mejora y ampliación de los conocimientos expuestos en la teoría ofrecida.
El formato vídeo es el recurso más utilizado en los MOOC para la transmisión de contenido teórico, pudiendo ser ofrecido en distintos modos (screencast, vídeo docente, etc.). También se utiliza el formato texto, pero se ubica como material complementario que se suele ofrecer a los usuarios para que puedan descargarlo y conservarlo en sus equipos.

Al final de cada bloque temático es frecuente que se proponga a los usuarios un ejercicio o examen parcial para comprobar los conocimientos adquiridos durante el tema. Su superación será crucial para poder acceder a realizar los exámenes finales.

La tabla 1 muestra un ejemplo sobre la forma de estructurar un tema o bloque temático. Se puede observar que para este ejemplo se ha dividido el tema 1 en dos unidades de trabajo: unidad 1.1 y unidad 1.2. Cada una de estas unidades tiene que contener un recurso, una actividad complementaria y material complementario.

El último bloque común en todos los cursos de Iversity es el examen final. Este bloque se sitúa al final de los bloques temáticos que componen el curso. Esta prueba de evaluación tiene como misión comprobar si el usuario ha adquirido los conocimientos que se han ofrecido en la acción formativa. Para ello, existen una serie de preguntas autocorregibles que hay que realizar en un tiempo limitado. Para obtener el certificado, tanto de asistencia como de superación, es indispensable superar este examen final.

\section{Tabla 1. Ejemplo de la estructura de un bloque temático en Iversity}

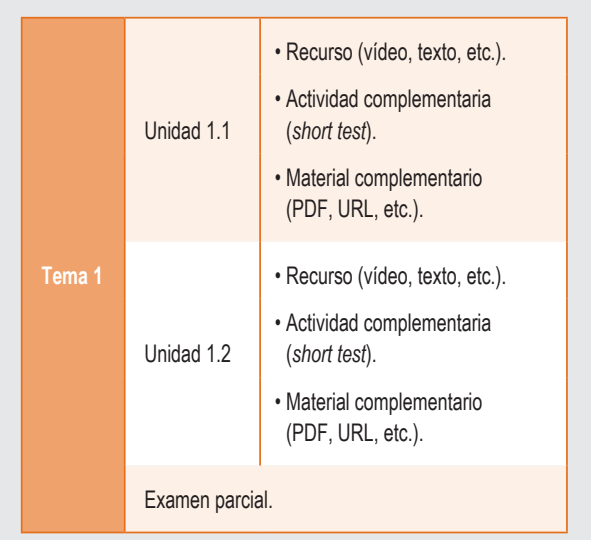

Fuente: elaboración propia 


\section{MOOC PARA LA ENSEÑANZA DEL ESPAÑOL COMO LENGUA EXTRANJERA}

La presente investigación se ha realizado bajo el marco del proyecto «Aplicación de las nuevas tecnologías a la enseñanza del español a través de cursos abiertos masivos online» (ANTEeCAMO). En este proyecto participaron investigadores de las áreas de ingeniería informática, idiomas y pedagogía.

\subsection{ANTEeCAMO}

El proyecto ANTEeCAMO se encuadra dentro de los proyectos de innovación docentes y ha supuesto el desarrollo de un MOOC entre el equipo de profesores de la UDIMA y expertos de Iversity. La temática que se ha desarrollado consiste en un curso para el aprendizaje de la lengua española en el nivel MCERL (marco común europeo de referencia para las lenguas: aprendizaje, enseñanza y evaluación) A1.1. Este tipo de cursos, en un nivel básico de aprendizaje, son poco comunes en el ámbito europeo, por lo que este proyecto ha constituido una piedra angular sobre la que apoyarse en futuros desarrollos.

Los principales objetivos desarrollados en este proyecto fueron:

- Diseñar un curso para el aprendizaje del español para un número grande de estudiantes.

- Estudiar y aplicar la metodología apropiada para diseñar un curso masivo online.

- Establecer un acuerdo de colaboración con expertos de la plataforma Iversity.

En el proyecto ANTEeCAMO se siguió una metodología en espiral, de forma que los resultados de una fase eran necesarios para iniciar la siguiente fase. El proyecto se planificó en siete fases. A continuación se describe de forma breve cada una de dichas fases:

- I fase. Se realizó el contacto con los expertos de Iversity con el objetivo de establecer la estructura inicial del curso.

- Il fase. Consistió en el análisis de la estructura inicial por parte de los expertos en idiomas y pedagogía. En esta fase se propusieron mejoras o modificaciones de la anterior estructura que fueron consensuadas con los expertos de Iversity.
- III fase. Consistió en la creación de contenidos educativos. Fue la más crítica del proyecto, ya que en ella se incluye la creación y la edición de los 23 vídeos de los que se compone el curso, así como la creación de más de 50 actividades y los correspondientes contenidos teóricos para cada unidad. En esta fase fue necesario la utilización de los medios audiovisuales de la UDIMA, así como la participación de actores para la realización de contenidos audiovisuales. Hay que destacar que para la creación de los vídeos de este curso hubo que recrear distintas situaciones y en distintos espacios o escenarios, por lo que se necesitaron ocho actores. Es importante señalar que no todos los actores participaron en los mismos escenarios, como habitualmente sucede en los MOOC. Una dificultad añadida radicó en que los vídeos se rodaron en espacios abiertos en el campus de la UDIMA y se utilizaron dos cámaras, así como el estudio de grabación de la UDIMA para la posterior edición de los vídeos. Además, también se introdujeron subtítulos en español para cada uno de los vídeos.

- IV fase. Consistió en la codificación de contenidos educativos y su implementación en la plataforma Iversity.

- V fase. Consistió en el lanzamiento y mantenimiento del MOOC. El lanzamiento se realizó dos meses antes de la apertura del curso y se llevaron a cabo acciones de marketing destinadas a conseguir estudiantes que estuvieran interesados en el curso. En este sentido, se mantuvieron reuniones con el Departamento de Publicidad y Relaciones Públicas de Iversity y con el Departamento de Marketing de la UDIMA.

- VI fase. Consistió en el análisis del impacto que originó el MOOC. Se considera que el $\mathrm{MOOC}$ ha causado un gran impacto. Ya en el inicio del curso de la primera edición había más de 5.800 estudiantes matriculados y, al final del curso, se registró un total de 13.076. Los estudiantes matriculados pertenecían a distintos países del mundo, como Alemania, Italia, EE. UU., India, China, Reino Unido, Ucrania, Rumanía, Pakistán, Turquía, Filipinas, Rusia, Canadá, Brasil y Egipto.

- VII fase. Consistió en la difusión de los resultados. En esta etapa se publicó un estudio preliminar del $\mathrm{MOOC}$ en el Congreso Internacional de Educación Abierta y Tecnología. 
Cabe destacar que en este proyecto se llevaron a cabo más de 35 reuniones. La mayoría de ellas fueron virtuales, a través de Blackboard Collaborate, Google Hangouts y Skype, y tuvieron lugar entre el equipo docente y los expertos de Iversity. También se realizaron reuniones entre el equipo docente y el Departamento de Idiomas, así como con el equipo técnico de grabación y edición de la UDIMA. Estos datos demuestran que este proyecto ha exigido un complejo trabajo de coordinación entre el Departamento de Idiomas, el Departamento de Innovación e Iversity, así como con el Departamento de Marketing y de Publicidad.

\subsection{Estructura del curso}

El MOOC diseñado tiene por título Spanish for Beginners ${ }^{14}$. Está dirigido claramente a un público internacional que busca aprender los fundamentos básicos del español, bien sea por interés personal o para aprender una nueva lengua de cara a sus estudios 0 a un trabajo.

El nivel del que se parte (A1.1) es el establecido por el MCERL, donde se define el progreso en el aprendizaje, describiendo lo que el estudiante puede hacer mediante el uso de la lengua. En concreto, el MOOC atiende a los siguientes descriptores correspondientes al nivel A1.1:

- Capacitar al estudiante para satisfacer las necesidades comunicativas más corrientes, a través de la interacción en diálogos, textos y discursos asequibles, en un registro estándar de formalidad e informalidad.

- Conocer y comprender las estructuras morfosintácticas básicas vinculadas a las funciones y a los contenidos propios de este nivel.

- Conocer y entender los contenidos de frases básicas para presentarse, saludar y despedirse.

- Ser capaz de pedir y dar información básica sobre personas y lugares de su entorno o imaginarios.

- Ser capaz de pedir algún objeto 0 asunto a alguien y de responder a peticiones.

- Entender y usar los elementos necesarios para realizar descripciones y presentaciones sencillas sobre sí mismo, otras personas y situaciones y lugares cotidianos.

14 URL del curso: https://iversity.org/en/courses/spanish-for-beginners
- Entender y realizar preguntas e instrucciones breves y sencillas que estén relacionadas con el espacio, con el tiempo y con temas de necesidades inmediatas o muy cotidianas.

- Entender y usar los procedimientos básicos para expresar los gustos, los intereses, los deseos y las sensaciones.

- Comprender informaciones, discursos, textos y mensajes muy breves y bien articulados, así como expresiones de uso frecuente.

- Conocer las estructuras básicas para escribir frases, oraciones y textos sencillos y muy concretos.

- Conocer y usar el léxico básico relativo a las situaciones concretas especificadas en los contenidos y en las funciones.

- Ser capaz de valorar la correcta adecuación pragmática en el empleo de las fórmulas de cortesía más sencillas y cotidianas relacionadas con los saludos, las despedidas y las presentaciones.

- Conocer y valorar las tradiciones y acontecimientos sociales y culturales más importantes del mundo hispano, asi como sus personajes más representativos.

El curso se ha estructurado en 20 lecciones prácticas, cada una con secuencias de vídeo, que resuelven situaciones reales de comunicación en diferentes contextos. El enfoque metodológico del que se parte trabaja los contenidos necesarios que llevan hacia la comunicación, ya que el objetivo final que se persigue es conseguir una competencia comunicativa que posibilite la interacción lingüística adecuada en las diferentes situaciones de comunicación. En el siguiente epígrafe se expone la estructura de este curso en Iversity, así como una explicación detallada de cada lección.

El objetivo general que se quiere cubrir con este curso es que el estudiante resuelva de forma práctica situaciones reales de la vida cotidiana y que, de esta forma, pueda adquirir una competencia comunicativa básica que le permita desenvolverse de forma fluida.

\subsection{Implementación del curso en Iversity}

El curso inicialmente estaba dividido en 5 bloques temáticos y cada uno de estos bloques en 4 unidades con distintos ejercicios y vídeos. En la tabla 2 se muestra el ejemplo del diseño inicial de un bloque temático, 
donde se puede observar que cada unidad se componía de 1 bloque de presentación y de otros 3 bloques. Hay que señalar que durante el montaje e implantación del curso en la plataforma se produjeron incidencias de carácter técnico que obligaron a cambiar el esquema inicial. Este tipo de estructura producía incidencias como las siguientes: velocidad de navegación inferior a la normal, bloqueo de la plataforma durante el montaje y edición del curso, imposibilidad de editar ciertos recursos que conformaban el curso, etc.

Esta limitación supuso un cambio en la estructuración del curso, pasando de 5 bloques temáticos principales, divididos en 4 unidades cada uno, a 20 bloques temáticos con sus respectivos recursos, como se observa en el ejemplo de la tabla 3.

\section{Tabla 2. Ejemplo de un bloque temático en el diseño inicial}

\begin{tabular}{|l|l|}
\hline Unidad 1.1. Presentación \\
$\cdot$ Unidad 1.1.1 \\
$\cdot$ Unidad 1.1.2 \\
$\cdot$ Unidad 1.1.3 \\
\begin{tabular}{|l} 
Examen parcial \\
Unidad 1.2. Presentación \\
$\cdot$ Unidad 1.2.1 \\
$\cdot$ Unidad 1.2.2 \\
$\cdot$ Unidad 1.2.3 \\
Examen parcial \\
Unidad 1.3. Presentación \\
$\cdot$ Unidad 1.3.1 \\
$\cdot$ Unidad 1.3.2 \\
• Unidad 1.3.3 \\
Examen parcial \\
Unidad 1.4. Presentación \\
$\cdot$ Unidad 1.4.1 \\
$\cdot$ Unidad 1.4.2 \\
$\cdot$ Unidad 1.4.3 \\
Examen parcial
\end{tabular} \\
\hline
\end{tabular}

Fuente: elaboración propia
El cambio de estructuración permitió reduci r el número de incidencias técnicas, que se estaban experimentando hasta el momento, aunque la velocidad de la plataforma, debido al tamaño y al peso del curso siguió siendo inferior a lo que se esperaba con el cambio estructural.

En las figuras 1 y 2 se pueden ver capturas de pantalla de la estructura del curso. En la figura 1 se muestra la estructura del tema 1 , mientras que en la figura 2 se muestra la estructura del tema 20 (último tema de este curso). Se puede observar que en la parte superior de la figura 1 se indica el nombre del curso (Spanish for Beginners), así como que el curso ha finalizado (Finished). En la parte izquierda

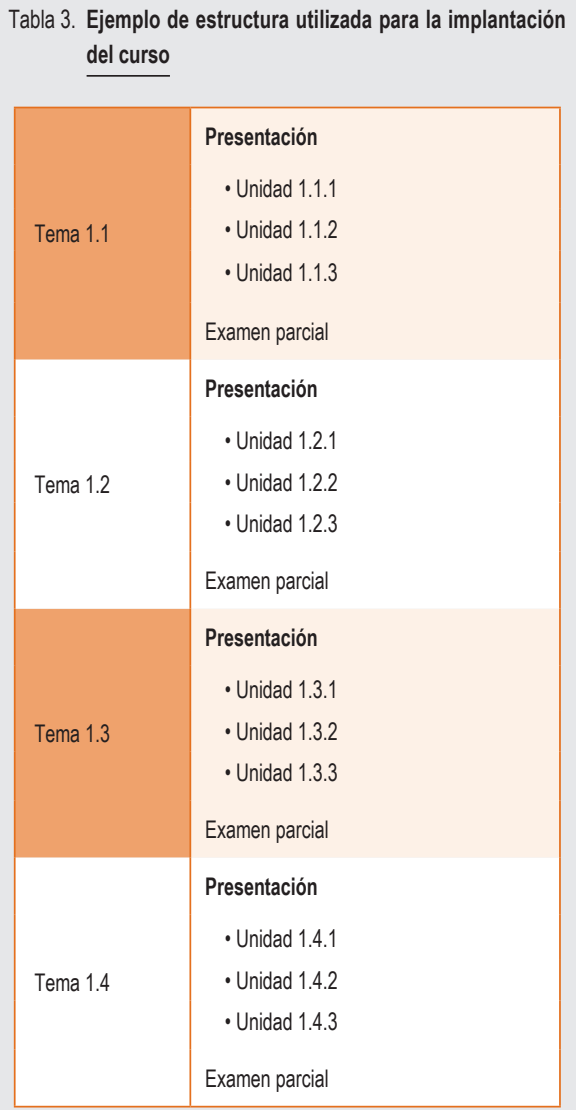

Fuente: elaboración propia. 
de ambas figuras se muestran los bloques temáticos (Chapter) y en la parte derecha se indican las unidades que componen un bloque temático y sus actividades y recursos asociados. Además, en la figura 2 se puede observar el examen final del curso, que se denota como CoA Exam. Este examen es el que se utiliza para valorar el aprendizaje final de los estudiantes y su superación da la posibilidad de solicitar un certificado de participación y un certificado de superación.

\section{Figura 1. Captura de pantalla de la estructura del tema 1}

\section{Spanish for Beginners rmast}

Chapters NAnnouncements Discussions 1 Course Info
$\begin{aligned} & \text { Chapter } 1 \text { Certificates } \\ & \text { EN CONTACTO CON EL ESPAÑOL I - Saludary } \\ & \text { presentarse }\end{aligned}$
$\begin{aligned} & \text { Chapter } 2 \\ & \text { EN CONTACTO CON EL ESPAÑOL III - Hablar de } \\ & \text { lenguas, preguntar por palabras desconocidas, } \\ & \text { mantener la conversación }\end{aligned}$
$\begin{aligned} & \text { Chapter } 3 \\ & \text { EN CONTACTO CON EL ESPAÑOL II - Preguntar y decir } \\ & \text { el lugar de origen, nacionalidad, Iugar de residencia }\end{aligned}$
$\begin{aligned} & \text { Chapter } 4 \\ & \text { EN CONTACO CON EL ESPAÑOL IV - Preguntar y decir } \\ & \text { para qué se estudia español, expresar finalidad, } \\ & \text { expresar intención }\end{aligned}$

\section{Figura 2. Captura de pantalla del último tema del curso}

Chapter 20

HÁBITOS PARA EL TIEMPO UBRE IV - Describir Y comparar actividades

CoA Exam

Start date: 16 Jun 10:01 (CEST) End dato: 01 Jul 23:59 (CEST)

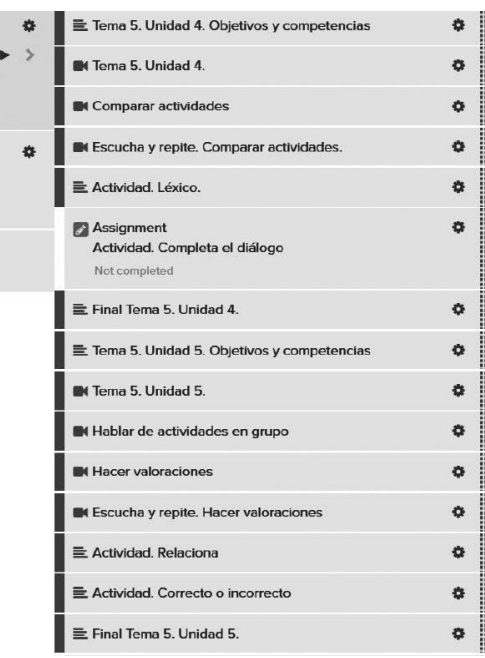

Fuente: captura del curso Spanish for Beginners en la plataforma Iversity. 
Teniendo en cuenta los problemas de diseño anteriores, a continuación se muestra un extracto de la estructura final del curso, donde se ha desarrollado con detalle el bloque temático 1. La estructura completa del curso puede consultarse en el apéndice 1. Para esta estructura se han utilizado las siguientes abreviaturas: bloque temático (BT), diálogo (D), examen final (EF), examen parcial (EP), homework (H), imagen (I), material adicional (MA), resumen de conceptos $(R)$, short test $(S T)$, texto $(T)$, vídeo $(\mathrm{V})$ y vídeo subtitulado (VS).

- BT0. Presentación del curso.

- BT1. En contacto con el español I. Saludar y presentarse.

- Tema 1. Presentación (V, ST, MA [T, D]).

- Saludar y presentarse (VS, MA [T, D, I]).

- Preguntar y decir el nombre (VS, MA [T, D, I]).

- Actividad. Deletrear (VS, MA [T, D, I]).

- Actividad. Escucha y repite. Deletrear (V).

- Actividad. Escucha y repite. Saludar y preguntar el nombre (V).

- Actividad. Completa (ST, MA [I]).

- Actividad. Relaciona (ST, MA [I]).

- Final tema 1. Unidad 1 (T, MA [R]).

- Actividad. Completa el diálogo (EP).

- BT2. En contacto con el español II. Hablar de lenguas, preguntar por palabras desconocidas, mantener la conversación.

- BT3. En contacto con el español III. Preguntar y decir el lugar de origen, nacionalidad, lugar de residencia.

- BT4. En contacto con el español IV. Preguntar y decir para qué se estudia español, expresar finalidad, expresar intención.

- BT5. Gente que conocemos I. Presentarse y describirse físicamente.

- BT6. Gente que conocemos II. Dar información sobre amigos y familiares.

- BT7. Gente que conocemos III. Hacer presentaciones sobre gente que conocemos, pedir y dar información sobre el estado físico.

- BT8. Gente que conocemos IV. Hablar de gustos y actividades, expresar deseos.
- BT9. El entorno, ciudades y barrios I. Hablar y preguntar por una dirección, hablar del origen y del destino.

- BT10. El entorno, ciudades y barrios II. Preguntar por una dirección, comprender instrucciones, indicar el camino y medios de transporte.

- BT11. El entorno, ciudades y barrios III. Expresar distancia. Expresar frecuencia.

- BT12. El entorno, ciudades y barrios IV. Preguntar y pedir información.

- BT13. Actividad social: comidas y bebidas I. Hablar de comidas y bebidas, expresar preferencias.

- BT14. Actividad social: comidas y bebidas II. Preguntar por un plato o una tapa, hablar de cantidades.

- BT15. Actividad social: comidas y bebidas III. Hablar de las horas y los momentos del día; comportamientos sociales.

- BT16. Actividad social: comidas y bebidas IV. Pedir la cuenta, hablar de costumbres.

- BT17. Hábitos para el tiempo libre I. Describir hábitos y costumbres en vacaciones.

- BT18. Hábitos para el tiempo libre II. Expresar gustos y preferencias, hacer una propuesta.

- BT19. Hábitos para el tiempo libre III. Recomendar lugares, hablar del tiempo.

- BT20. Hábitos para el tiempo libre IV. Describir y comparar actividades.

- EF.

Se puede observar que estructuralmente el curso se dividió en 20 bloques temáticos y en 2 bloques adicionales, correspondientes al bloque de presentación del curso y al bloque del examen final. En este esquema se ha desarrollado de forma detallada el bloque temático 1 (BT1. En contacto con el español I. Saludar y presentarse), que se ha dividido en 10 unidades de trabajo. En la estructura de cada bloque temático se presenta el título de la unidad seguido de una serie de abreviaciones entre paréntesis, que indican los recursos utilizados en la unidad.

Por ejemplo, la primera unidad de este bloque es la presentación del bloque temático, que contiene un vídeo (V) de una oficina Erasmus en la que dos estudiantes se presentan y preguntan a la coordinadora Erasmus; una actividad de tipo short test (ST), 
que contiene preguntas sobre el vídeo; y material adicional (MA), consistente en los objetivos de la unidad en formato texto $(T)$ y en el diálogo del vídeo (D) en formato PDF. La segunda unidad de este bloque está representada de esta forma: «Saludar y presentarse (VS, MA [T, D, I])», lo que significa que el título de la unidad es «Saludar y presentarse» y consta de un vídeo subtitulado (VS) y de material adicional (MA), consistente en el diálogo en formato texto $(T, D)$ y en una serie de contenidos gramaticales en formato imagen (I). La penúltima unidad de este bloque, «Final Tema 1. Unidad 1 (T, MA [R])», corresponde al final del tema 1 y consta de un resumen de los conocimientos adquiridos en formato texto $(\mathrm{T})$ y de material adicional (MA), consistente en un resumen de los conceptos (R) tratados en el bloque temático en formato PDF. La última unidad de este bloque consiste en el examen parcial (EP) del bloque temático 1. Hay que señalar que cada vídeo de cada bloque temático se divide en fragmentos que después se utilizan en las unidades que contiene este bloque temático. La estructura seguida en el resto de los bloques temáticos es muy similar a la del bloque 1, pero añadiendo o modificando las actividades, de forma que se consiga un dinamismo en el aprendizaje del estudiante.

Como se ha señalado anteriormente, dentro de cada bloque temático se sigue el siguiente esquema formado por cuatro fases de aprendizaje:

\section{A) Fase de presentación}

- En contexto. Secuencia de vídeo. El vídeo es la muestra de lengua real que presentará, de forma contextualizada, los recursos lingüísticos y funcionales que los alumnos necesitarán para adquirir la competencia comunicativa básica que les permita comunicarse en la lengua que se estudia en diferentes situaciones de la vida real.

- Objetivo de esta fase. Potenciar desde la muestra de lengua los recursos necesarios para adquirir competencia comunicativa.

Las figuras 3 y 4 muestran dos vídeos de los bloques temáticos 1 y 20, respectivamente. Se puede observar cómo en ambas se ofrece al estudiante un esquema en español y en inglés de lo que se va a tratar en cada vídeo. Actualmente se está trabajando para incluir subtítulos en otros idiomas (chino, ruso, alemán, francés, etc.), dada la demanda desde su primera implementación y como estrategia de aprendizaje, ya que en niveles básicos (como en el que trabajamos, A1.1) es normal que aparezcan las instrucciones en la lengua materna del discente. Esto va a influir positivamente en el acercamiento que este haga hacia la lengua que aprende, dotándole de seguridad y autonomía como clave metodológica. Como se comentaba anteriormente, la participación es indispensable para aprender en estos entornos virtuales, pero hay otros factores que se deben tener en cuenta para que el alumno no abandone y favorezca su autodisciplina: motivación, actitud, competencia tecnológica, etc.

Figura 3. Muestra de vídeo de la unidad 2 del bloque temático 1

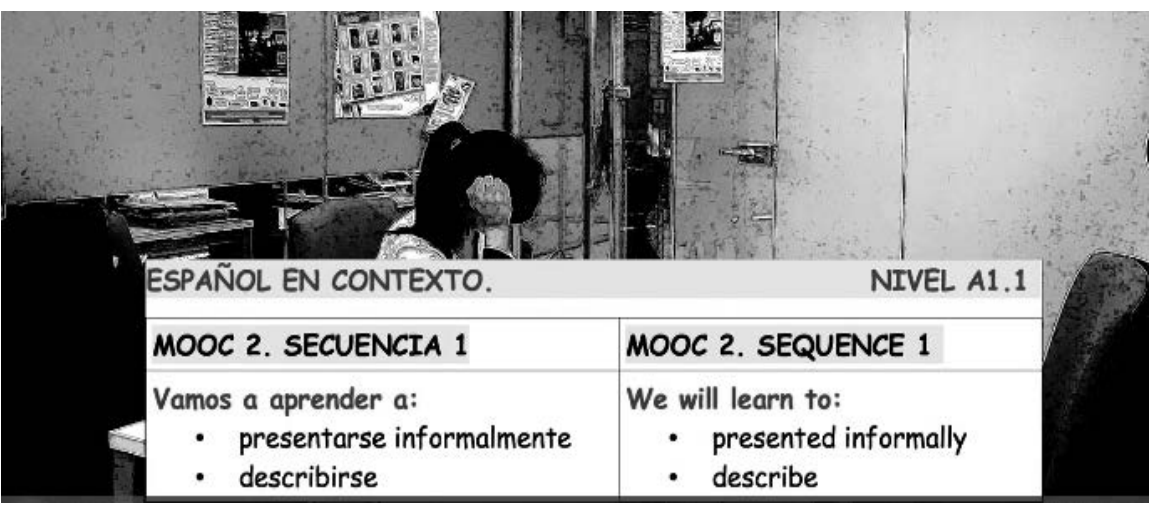

Fuente: captura del curso Spanish for Beginners en la plataforma Iversity. 


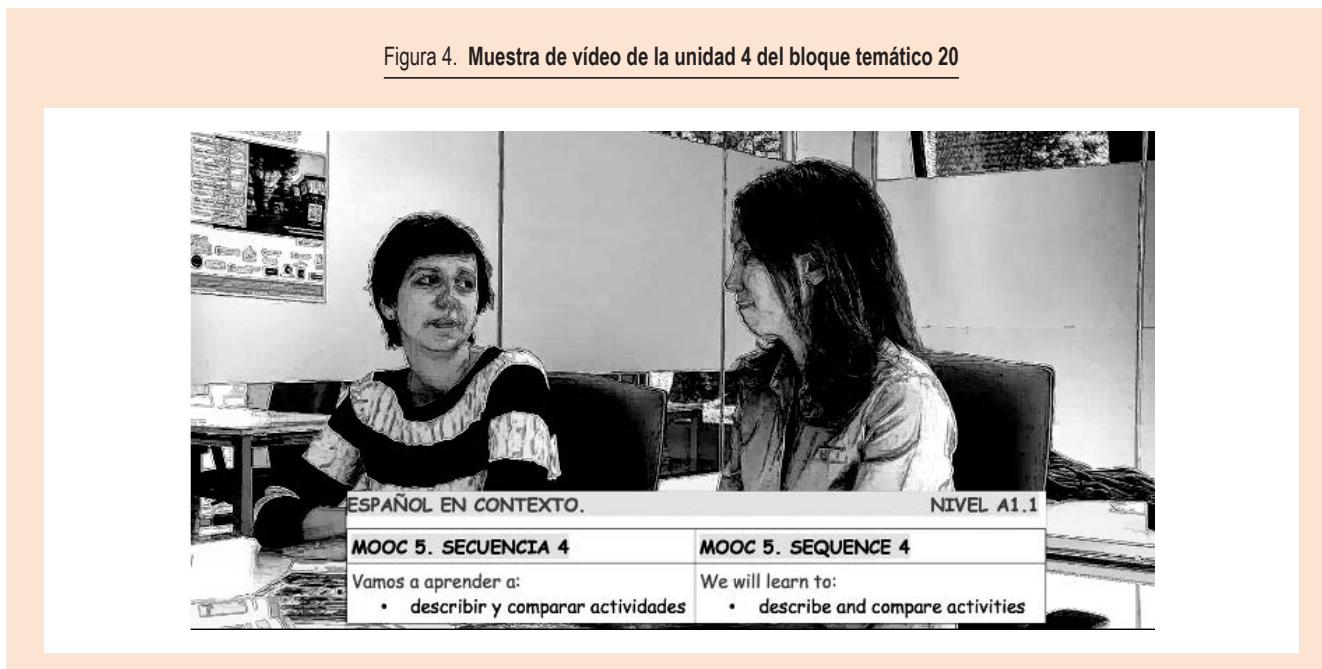

Fuente: captura del curso Spanish for Beginners en la plataforma Iversity.

B) Fase de observación

- Lengua y comunicación. Desde la muestra de lengua (vídeo) se observarán las distintas formas y recursos que han ido apareciendo a lo largo del visionado y que los alumnos necesitarán para llevar a la práctica lo aprendido u observado.
- Objetivo de esta fase. Preparar al alumno para la comunicación.

En la figura 5 se observa una imagen en la que el estudiante puede ejercitar la práctica mediante los subtítulos del diálogo que se muestran en el vídeo.



Fuente: captura del curso Spanish for Beginners en la plataforma Iversity. 
C) Fase de ejercitación y desarrollo competencial

- Tipología de actividades. Para poner en práctica las diferentes situaciones de comunicación que se han visionado, el estudiante encontrará una amplia gama de actividades que le ayudarán a ejercitar estas situaciones e integrar destrezas.

- Objetivo de esta fase. Interiorizar los recursos lingüísticos necesarios a través de la práctica funcional y formal.

En la figura 6 se muestra el tipo de actividad autocorregible short test. Se observa que el estudiante puede responder a la pregunta y al mismo tiempo visualizar el vídeo. Esta es una característica importante para reforzar el aprendizaje de los estudiantes.

D) Fase de comprobación

- Autoevaluación. El estudiante siempre tendrá la opción de comprobar lo que ha aprendido mediante una autoevaluación. Se le presentan los conocimientos adquiridos en modo formato texto, como se puede observar en la figura 7 .

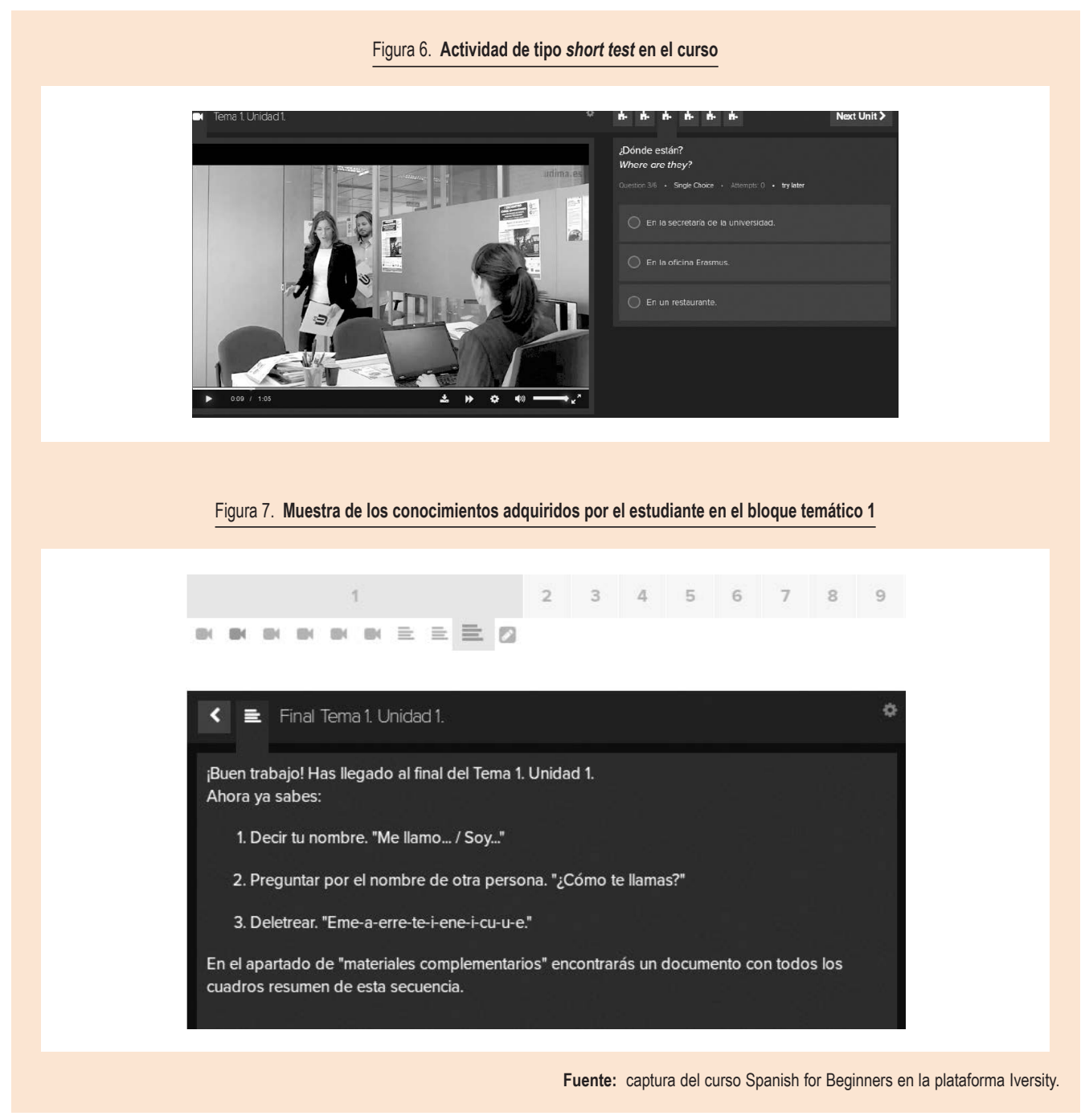




\subsection{Análisis y valoración del MOOC Spanish for Beginners}

La base de fundamentación principal ha sido el MCERL, desde donde se han diseñado las competencias lingüísticas, pragmáticas y socioculturales e interculturales. En este sentido, seguir las orientaciones del MCERL hace que se considere al alumno como un miembro de una sociedad en la que, movilizando sus competencias, puede llevar a cabo actividades y tareas lingüísticas y no lingüísticas.

Desde la metodología ecléctica a la que se ha vinculado el MOOC Spanish for Beginners, se concibe este como un espacio de comunicación (por las muestras de lengua reales en el que se inscribe cada uno de los vídeos), un espacio de aprendizaje (por las actividades propuestas) y un espacio de reflexión sobre la lengua, su uso y aprendizaje (adecuado en cada momento al nivel de conocimientos de español, para que los alumnos puedan ser capaces de actuar). Los espacios descritos pretenden desarrollar en el alumno no solo las competencias comunicativas (lingüísticas, pragmáticas y socioculturales), sino también las competencias generales (conocimientos, destrezas y habilidades lingüísticas y capacidad para aprender). De este modo, creemos que mediante una metodología ecléctica, tecnológica y pedagógicamente hablando, como la que se ha descrito, es difícil reconocer los avances tecnológicos para la enseñanza, pero consideramos que los docentes de segundas lenguas deben tener en cuenta que las situaciones de enseñanza responden a características que no pueden generalizarse y, más aún, cuando el curso MOOC está dirigido a un público internacional.

La presentación del contenido se hace de manera situacional (de uso) y funcional (pragmática), estableciendo, de esta manera, una secuencia que favorece el aprendizaje desde la misma metodología y a través del pulso tecnológico, por lo que la clave de presentación del contenido huye de lo gramatical y se prioriza el desarrollo de competencias comunicativas a través de situaciones reales de uso de la lengua.

Los recursos didácticos que se aportan son principalmente material audiovisual, audio y material escrito. Consideramos que el desarrollo de recursos didácticos viene determinado por el marco en el que se ins- cribe el curso, haciendo que se «desmorone» el pulso entre tecnología y enseñanza.

El desarrollo de la competencia comunicativa conseguida por el estudiante desde sus distintos componentes (lingüístico, pragmático y sociocultural) se consigue con la adquisición de contenidos nociofuncionales, es decir, haciendo hincapié en el uso de la lengua con un fin comunicativo. Por lo tanto, desarrollar la competencia comunicativa sobre la adquisición de contenido funcional implica el abandono del modelo tradicional de enseñanza cuyo desarrollo competencial se adquiría sobre la adquisición de contenidos gramaticales.

Respecto a la interculturalidad, al dirigirse el MOOC a un público internacional, se ha marcado como eje de este el aprendizaje intercultural, fomentando la construcción de conocimiento desde él mismo. Todas las secuencias de vídeo invitan al estudiante a conocer y valorar las tradiciones y los aspectos sociales y culturales más importantes del mundo hispano. Los propios protagonistas de las secuencias de vídeo fomentan lazos de comunicación interpersonal e intercultural. Así, desde el aprendizaje intercultural se garantiza el acceso al conocimiento sociocultural del mundo hispano, intentando que perciba la diversidad cultural y lingüística que hay en él, además de favorecer el desarrollo de la competencia intercultural.

La interacción, gestionada desde la destreza escrita, ha circunscrito el enfoque metodológico al desarrollo de destrezas que tienen que ver con lo tradicional: leer, escuchar, comprender y escribir. De este modo, la interacción ha venido determinada por las características del entorno formativo (plataforma Iversity), lo que invita a la reflexión sobre el nuevo entorno y sus posibilidades, así como a redefinir un modelo metodológico que pueda adaptarse al MOOC

El sistema de autoevaluación y evaluación del aprendizaje se resuelve con el uso de elementos no verbales (sonidos y emoticonos) que invitan al alumno a participar y/o seguir participando, evitando interpretar texto escrito. Con la autoevaluación al final de cada capítulo se tiene la opción de comprobar lo que ha aprendido el alumno, además de provocar la reflexión del mismo sobre su aprendizaje. De esta forma se consigue que el estudiante sea más consciente de su aprendizaje. 


\section{RESULTADOS}

El MOOC se ofreció en dos ediciones, una por cada año académico. La primera edición del MOOC fue seguido por 13.076 estudiantes, de los cuales el 2\% (351 estudiantes) obtuvo un certificado de participación, y de estos solo el $2 \%$ logró un certificado de superación. En su segunda edición, el MOOC fue seguido por 5.923 estudiantes con tasas similares de participación. A continuación se describe la evolución de la matriculación, distribución por países, género y edad por cada edición.

\subsection{Evolución de la matriculación y distri- bución por países}

En la parte superior de la figura 8 se muestra la evolución de la matriculación del MOOC en su primera edición, desde dos meses antes de su lanzamiento hasta la finalización del curso. Los datos mostrados corresponden a las nuevas matrículas en un periodo de tiempo determinado. Por ejemplo, el 5 de febrero de 2015, con el inicio de la campaña de marketing, se matricularon 772 estudiantes, pero en el momento del lanzamiento había más de 6.800 estudiantes matriculados. En la parte inferior de la figura 8 , se puede observar el porcentaje de estudiantes según los países de origen. Entre los países de mayor índice de matriculación están Alemania, Reino Unido e India. En este gráfico se observa que el $43,4 \%$ de los estudiantes pertenecía a "otro país», es decir, estos estudiantes no especificaron el país de origen.

\section{Figura 8. Estadísticas de matriculación y distribución por países en la primera edición del curso}

Total number of enrolled students: 13.076

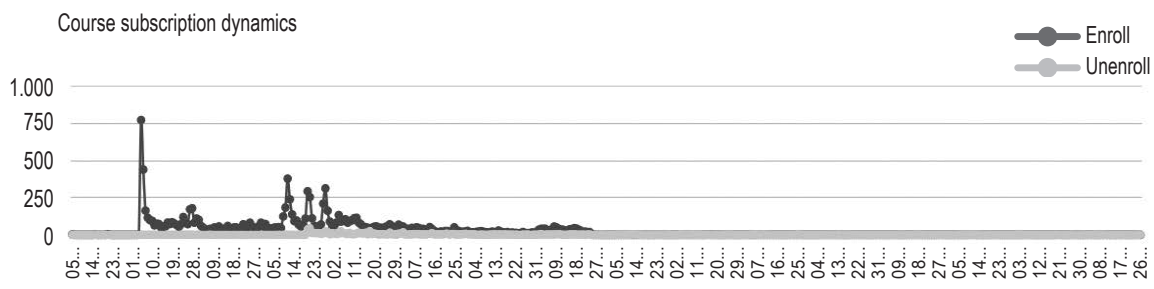

Geography

Top countries

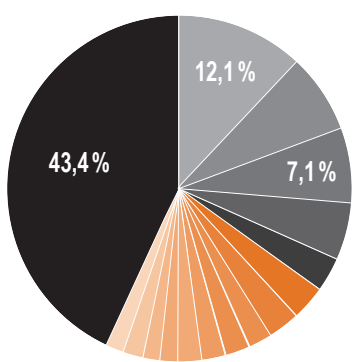

Top cities
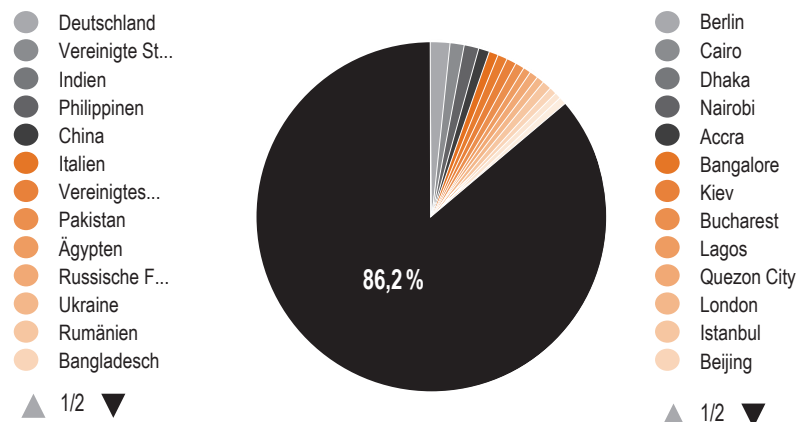

$\Delta 1 / 2$ 
En la figura 9 se puede observar que el número total de estudiantes matriculados en la segunda edición de este MOOC fue de 5.923. Las estadísticas muestran una distribución por países y ciudades muy similar a las de la primera edición.

\subsection{Distribución por género y edad}

Las figuras 10 y 11 muestran los estudiantes clasificados por edad en ambas ediciones. En el gráfico izquierdo de la figura 10 , se observa que un $65 \%$ de los estudiantes no concretaron su género y, de los que sí lo especificaron, hubo el mismo número de mujeres que de hombres matriculados. En el caso de la edad, la gran mayoría de los estudiantes tenían más de 22 años y menos de 42 (véase gráfico derecho de la figura 10), pero esta estadística debe ser estudiada con cautela, ya que el $84 \%$ de los estudiantes no especificaron la edad. Las estadísticas de la edad para la segunda edición muestran resultados similares, pero se observa un ligero aumento del número de estudiantes, con edades comprendidas entre los 19 y los 21 años.

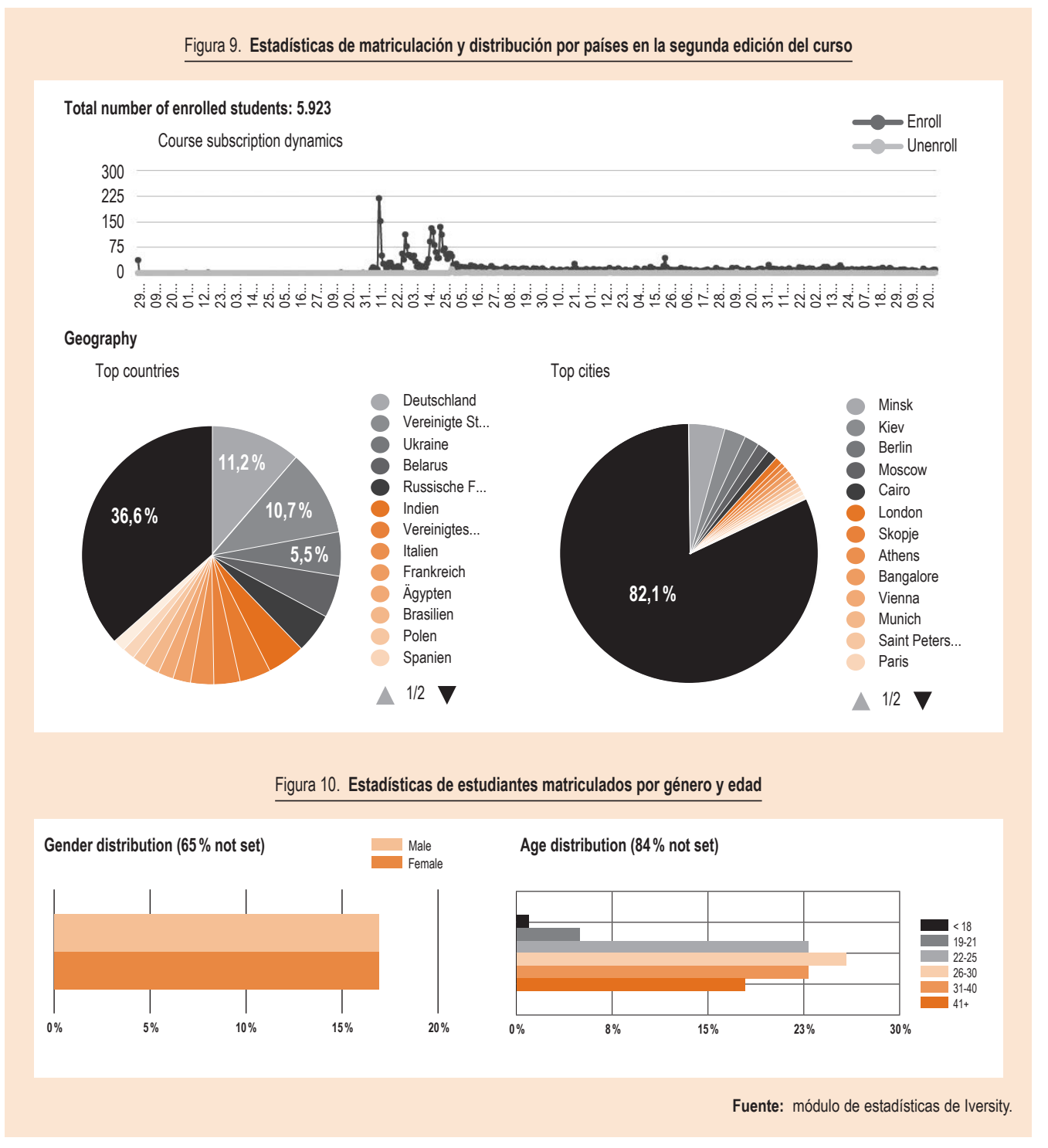


Figura 11. Estadísticas de estudiantes matriculados por edad

\section{Age distribution ( $86 \%$ not set)}

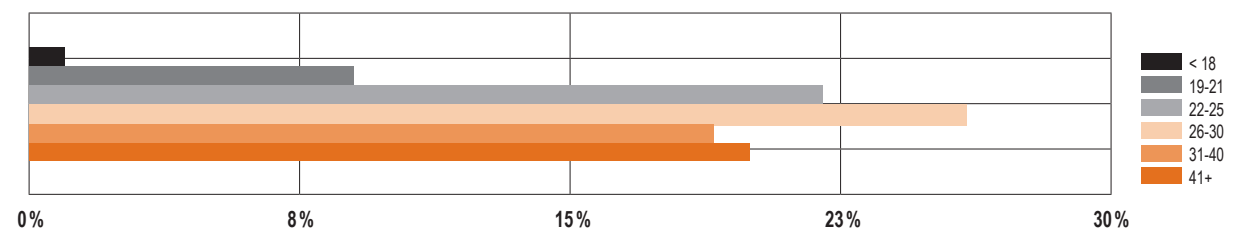

Fuente: módulo de estadísticas de Iversity

\subsection{Análisis de la encuesta}

La encuesta desarrollada se diseñó para conocer las opiniones de los estudiantes sobre el curso desarrollado en la primera edición del MOOC. Con el objetivo de conseguir un mayor número de respuestas, la encuesta se diseñó con 10 preguntas en una escala de Likert de 1 a 5 , donde 1 indicaba un total desacuerdo y 5 un total acuerdo. Las preguntas eran relativas a la satisfacción del curso, si las actividades eran apropiadas y estaban correctamente explicadas, si el número de actividades era excesivo, si habían surgido problemas en determinados tipos de actividades, la opinión sobre los vídeos y la utilización de subtítulos y si el curso debería estar también en inglés.

Los resultados de la encuesta mostraron que 95 estudiantes respondieron a la encuesta, lo que supone una participación de un $27 \%$ sobre los estudiantes que finalizaron el curso.

Cabe destacar que más del $60 \%$ de los estudiantes estuvieron de acuerdo en que se habían divertido con el curso, aunque un $9,5 \%$ se mostraron muy en desacuerdo. No obstante, un $34,7 \%$ coincidieron en que las actividades no estaban bien explicadas, frente a un $50,5 \%$ que pensaron que si estaban bien explicadas. En este sentido, un 65,3\% estuvieron de acuerdo en que el inglés podría ayudar a mejorar sus resultados en el curso. Respecto a los vídeos, los resultados mostraron que un $64,3 \%$ consideraron que los vídeos eran apropiados para el curso y $74,8 \%$ manifestaron que los subtítulos en los vídeos les habían ayudado a comprender los contenidos del curso. Con respecto a las actividades, un $64,2 \%$ estuvo a favor de las actividades tipo homework, pero un $50,5 \%$ de los estudiantes manifestaron tener problemas con este tipo de actividades.

\section{DISCUSIÓN}

La creación de un MOOC supone un gran reto para el equipo docente, y este reto es aún mayor cuando este MOOC está orientado a enseñar una lengua. Hay dos aspectos clave que es importante contemplar en el momento de realizar un proyecto como el expuesto. El primero es tener en cuenta que en los $\mathrm{MOOC}$ necesitamos un equipo docente formado por instructores, creadores de contenidos, curadores, pero también es necesario un equipo técnico, por lo que es necesario que la dirección de la institución esté en correcta sintonía con el equipo docente, ya que un gran número de personas de la institución participarán de manera directa o indirecta en la creación de este MOOC. EI segundo aspecto importante es el conocimiento completo de la plataforma en la que vayamos a alojar el MOOC, así como la interacción y comunicación continua con un equipo de la plataforma. En este trabajo utilizamos la plataforma Iversity, que está creada para el alojamiento de cursos pequeños y breves, lo que supuso un problema a la hora de diseñar y crear el curso Spanish for Beginners, ya que contenía un gran número de recursos didácticos. Esto se tradujo en pro- 
blemas técnicos en el montaje del curso, puesto que esta plataforma no soportaba tanto peso, y se ralentizaba la navegación. Otro problema que no hay que olvidar es que el aprendizaje del español exige disponer en el teclado de caracteres inexistentes en teclados de otros países. Por ejemplo, la acentuación lingüística española y letras como la «ñ» no se encontraban en la mayoría de los teclados extranjeros, lo que supuso un inconveniente a la hora de la realización de los ejercicios propuestos. No obstante, se les ofreció a los estudiantes la alternativa de generar estos caracteres a través del foro de discusión. Otra dificultad añadida fue que la tipología de actividades que se propone en Iversity no era suficiente para el curso diseñado y hubo que adaptar las actividades a las de Iversity. Por ejemplo, las actividades de emparejamiento fueron adaptadas. Esta dificultad ha de tenerse en cuenta en el diseño inicial del curso, ya que este debe estar adaptado a las posibilidades que ofrece la plataforma.

Otro de los aspectos clave en un MOOC es el análisis de los resultados obtenidos, es decir, el análisis de las interacciones de los estudiantes, del progreso, del proceso de aprendizaje, de la didáctica seguida, de los posibles errores de diseño, etc. Para este cometido, Iversity proporciona una ventana de estadísticas (Administration $\rightarrow$ Statistics), desde la que están disponibles las estadísticas de la evolución en la matriculación, distribución de países y ciudades, número de estudiantes que han comenzado una unidad, distribución por géneros y por edades. Estas estadísticas no son suficientes para realizar un análisis completo del MOOC, ya que no resultan fácilmente exportables y la interfaz no da la posibilidad de agrupar los datos en función de determinadas variables como la fecha o la actividad. Además, los estudiantes matriculados en Iversity no tienen obligación de especificar datos personales, como el país de origen, su género o edad. En la primera edición de este MOOC, el $44 \%$ de los estudiantes no indicó el país de origen, el $65 \%$ no indicó su género y el $84 \%$ no incluyó su edad. Por lo tanto, los datos mostrados en el epígrafe anterior deben ser interpretados teniendo en cuenta esta consideración.

Respecto a la evolución del número de matriculados, este MOOC ha supuesto un importante impacto, ya que en su primera edición más de 13.000 usuarios manifestaron su interés por el curso y se matricularon en él, y en su segunda edición hubo cerca de 6.000 estudiantes matriculados. Este hecho demuestra que existe un gran interés en aprender la lengua española mediante los MOOC, ya que el curso fue seguido por más de 19.000 estudiantes en los dos años que se ha lanzado. Además, este curso permaneció durante varias semanas en los primeros puestos del ranking que elabora Iversity sobre los cursos con más matriculados. No obstante, debido a problemas técnicos y a mejoras en el diseño del curso, solo un $2 \%$ de los estudiantes terminaron el curso de forma satisfactoria. Este dato no debe ser interpretado como poco favorable, ya que estos estudiantes mostraron un gran interés por el curso y quedaron muy satisfechos.

Con el objetivo de valorar los aspectos que había que mejorar en relación a la primera edición, se realizó una encuesta a los estudiantes que siguieron el curso hasta el examen final. En esta encuesta, los estudiantes mostraron los aspectos que ellos consideraban que había que mejorar en la nueva edición, y que se tuvieron en cuenta para el diseño del MOOC Spanish for Beginners (segunda edición), en la que se añadieron subtitulos y explicaciones de las actividades en idioma inglés y se contó con la participación de ayudantes de los profesores para dinamizar los foros.

\section{CONCLUSIONES Y RETOS}

En este trabajo se ha presentado de forma exhaustiva el diseño, la creación y la ejecución de un MOOC para aprender idiomas. El MOOC se alojó en la plataforma Iversity y en sus dos ediciones consiguió una matriculación de más de 19.000 estudiantes. Se ha demostrado que los MOOC que se utilizan para aprender una lengua extranjera se encuentran en una primera fase y que existe relativamente un número menor que en otras disciplinas.

Aunque los MOOC se llevan desarrollando desde el año 2008, aún quedan preguntas por responder, desde su filosofía estratégica (formación masiva, en formato abierto, online y promoción de cursos), pasado por la calidad y el reconocimiento de los mismos, la identificación del estudiante, la licencia de contenidos y un modelo económico sostenible, hasta la política educativa que se pretende desarrollar. En este trabajo se ha subrayado que el diseño metodológico viene determinado por la plataforma, por lo que a veces resulta difícil reconocer los avances tecnológicos para la enseñanza. A pesar de ello, los 
resultados mostrados nos permiten confirmar que estos entornos ofrecen las competencias necesarias para poner en funcionamiento programas de aprendizaje de lenguas extranjeras. Tal vez la clave para empezar a despejar incógnitas y resolver el binomio tecnología-pedagogía sea reflexionar sobre los indicadores de calidad de esta nueva modalidad e-learning, tales como la planificación, el diseño, la tutorización y la interacción.

Desde el MOOC propuesto, Spanish for Beginners, se abre una nueva vía para la investigación que tiene que ver con la necesidad de crear, en este nuevo

\section{BIBLIOGRAFÍA}

Aleven, V.; McLaren, B.; Sewall, J. y Koedinger, K. [2006]: «The cognitive tutor authoring tools (CTAT): preliminary evaluation of efficiency gains», Proceedings of Intelligent Tutoring Systems, Lecture Notes in Computer Science, 4.043, Jhongli (Taiwan): Springer Berlin Heidelberg, págs. 61-70.

Bates, A. W. [1995]: Technology open learning and distance education, London/NewYork: Routledge.

Bautista, G.; Borges, F. y Forés, A. [2006]: Didáctica universitaria de entornos virtuales de enseñanza-aprendizaje, Madrid: Narcea.

Brusilovsky, P. [1996]: «Methods and techniques of adaptive hypermedia», User Modeling and User-Adapted Interaction: The Journal of Personalization Research, 6 (2-3), págs. 87-129

Cabero, J. [2015]: «Reflexiones educativas sobre las tecnologías de la información y la comunicación (TIC)», Tecnología, Ciencia y Educación. CEF, 1, pág. 26.

Carro, R. M.; Pulido, E. y Rodríguez, P. [1999]: «Dynamic generation of adaptive internet-based courses», Journal of Network and Computer Applications, 22, págs. 249-257.

Chang, C. [2014]: Iversity plots a new path for european MOOCs. Technical Report. Disponible en: https:// www.class-central.com/reportiversity-european-moocs/ [Consultado: mayo de 2017].

Chen, F.; Myers, B. y Yaron, D. [2000]: Using handheld devices for tests in classes. Technical Report. CMU-CS00-152. Human Computer Interaction Institute. entorno e-learning, un currículo capaz de generar el cambio metodológico y de política educativa. Es necesario abrir líneas de investigación metodológicas en lo que se refiere a los contenidos de aprendizaje. El profesor ha de investigar nuevas líneas de acercamiento a los contenidos de forma significativa. La incorporación de la TIC en las aulas de lenguas extranjeras como nuevas herramientas de aprendizaje, de conocimiento y de desarrollo intelectual es el pilar fundamental de la formación tanto para el profesorado como para el discente.

Daniel, J. [2012]: «Making sense of MOOCs: musings in a maze of myth, paradox and possibility", Journal of Interactive Media in Education, 3, artículo 18.

Downes, S. [2007]: «What connectivism is», Connectivism Conference Forum, Manitoba (Canada): University of Manitoba.

Fernández-Pampillón, A. [2009]. «Las plataformas e-learning para la enseñanza y el aprendizaje universitario en internet», en C. López Alonso y M. ${ }^{a}$ Matesanz del Barrio (eds.), Las plataformas de aprendizaje: del mito a la realidad, Madrid: Biblioteca Nueva, págs. 45-47.

Gee, J. P. [2010]: New digital media and learning as an emerging area and "worked examples» as one way forward, Cambridge (MA): The MIT Press.

Gee, J. y Hayes, E. R. [2011]: Learning and language in the digital age, New York: Routledge.

Gros, B. [2002]: «Constructivismo y diseños de entornos virtuales de aprendizaje», Revista de Educación, 328, págs. 225-247.

Hafez, K. [2011]: «Global journalism for global governance?», Theoretical Visions, Practical Constraints. Journalism, 12 (4), págs. 483-496.

Iversity [2015]: Conceptual design guidelines for MOOCs on the Iversity platform. Techical Report.

Jenkins, H. [2009]: Fans, bloggers y videojuegos: la cultura de la colaboración, Barcelona: Paidós Ibérica.

Landeta, A. [2012]: Global e-learning, Madrid: CEF.

Larsen-Freeman, D. y Long, M. H. [1991]: An introduction to second language acquisition research, New York: Routledge. 
Matterlart, A. [2007]: Historia de la sociedad de la información, Barcelona: Bolsillo Paidós.

McLuhan, M. [1996]: Comprender los medios de comunicación: las extensiones del ser humano, Barcelona: Paidós Comunicación.

Moral, M. ${ }^{a}$ E. del y Villalustre, L. [2005]: «Adaptación de los entornos virtuales a los estilos cognitivos de los estudiantes: un factor de calidad en la docencia virtual», Pixel-Bit, 26, págs. 17-25.

Open Education Europa [2016]: Open education scoreboard. Technical Report. European Commission.

Pérez y Pérez, R. [2007]: «Employing emotions to drive plot generation in a computer-based storyteller», Cognitive Systems Research, 8 (2), págs. 89-109.

Regalado, A. [2 de noviembre de 2012]: «The most important education technology in 200 years», MIT Technology Review.
Roig, J. y Fernández, S. [2015]: «Un nuevo modelo de e-learning en el panorama educativo actual», Tecnología, Ciencia y Educación. CEF, 1, págs. 47-51.

Salvi, P. y Bravo, J. [2013]: «A comprehensive overview of MOOCs: antecedents and successful developments», en G. J. Palazio (ed.), Proceedings of Ikasnabar 2013, the 6th International Conference on Open Education and Technology (MOOCs, PLEs and eLearning platforms), Bilbao: Universidad del País Vasco, págs. 255-268.

Sosnovsky, S. y Brusilovsky, P. [2005]: «Layered evaluation of topic-based adaptation to student knowledge», Proceedings of 4th Workshop on the Evaluation of Adaptive Systems at 10th International User Modeling Conference, Edinburgh, págs. 47-56.

Thommen, B. [2008]: «Social knowledge and social representations: boon and bane of complex integrative concepts», Culture \& Psychology, 14 (2), págs. 245-252.

\section{APÉNDICES}

En esta parte del documento se incluye la estructura completa del curso y la encuesta realizada a los estudiantes.

\section{Estructura completa del curso}

Para esta estructura se han utilizado las siguientes abreviaciones: bloque temático (BT), diálogo (D), examen final $(E F)$, examen parcial $(E P)$, homework $(H)$, imagen (I), material adicional $(M A)$, resumen de conceptos $(R)$, short test $(\mathrm{ST})$, texto $(\mathrm{T})$, vídeo $(\mathrm{V})$ y vídeo subtitulado (VS).

BTO. Presentación del curso.

BT1. En contacto con el español I. Saludar y presentarse.

- Tema 1. Unidad 1. Presentación (V, ST, MA [T, D]).

- Saludar y presentarse (VS, MA [T, D, I]).

- Preguntar y decir el nombre (VS, MA [T, D, I]).

- Actividad. Deletrear (VS, MA [D, T, I]).

- Actividad. Escucha y repite. Deletrear (V). 
- Actividad. Escucha y repite. Saludar y preguntar el nombre (V).

- Actividad. Completa (ST, MA [I]).

- Actividad. Relaciona (ST, MA [I]).

- Final Tema 1. Unidad 1 (T, MA [R]).

- Actividad. Completa el diálogo (EP).

BT2. En contacto con el español II. Preguntar y decir el lugar de origen, nacionalidad, lugar de residencia.

- Tema 1. Unidad 2. Presentación (V, ST, MA [T, D]).

- Preguntar y decir el lugar de residencia (VS, MA [T, D, I]).

- Actividad. ¿Conoces estos países? (ST [I]).

- Actividad. Escucha y repite. Nacionalidades (V).

- Actividad. Masculino y femenino (ST [I])

- Actividad. Escucha y repite. Adjetivos en plural (V).

- Actividad. Formación del plural (ST [I]).

- Actividad. ¿De dónde son? (ST [I]).

- Actividad. ¿Dónde vives? (ST [I]).

- Actividad. Práctica con interrogativos (ST, MA [I]).

- Final Tema 1. Unidad 2 (T, MA [R]).

- Actividad. Escucha y completa el diálogo (EP).

BT3. En contacto con el español III. Hablar de lenguas, preguntar por palabras desconocidas, mantener la conversación.

- Tema 1. Unidad 3. Presentación (V, ST, MA [T, D]).

- Hablar de lenguas (VS, MA [T, D, I]).

- Preguntar por palabras desconocidas y por cosas que no se entienden (VS, MA [T, D, I]).

- Actividad. Relaciona las preguntas con las respuestas (ST).

- Actividad. Adverbios (ST, MA [I]).

- Actividad. Ordena las frases (ST [I]).

- Actividad. Verbos (-ar) (ST, MA [I]).

- Final Tema 1. Unidad 3 (T, MA [R]).

- Actividad. Completa el diálogo (EP).

BT4. En contacto con el español IV. Preguntar y decir para qué se estudia español, expresar finalidad, expresar intención.

- Tema 1. Unidad 4. Presentación (V, ST, MA[T, D]).

- Preguntar y decir por qué se habla español (VS, MA [T, D, I]).

- Actividad. Por, para y porque (ST, MA [I]).

- Actividad. Orden de frases (ST [I]).

- Final Tema 1. Unidad 4 (T, MA [R]).

- Actividad. Completa el diálogo (EP). 
BT5. Gente que conocemos I. Presentarse y describirse físicamente.

- Tema 2. Unidad 1. Objetivos y competencias (T).

- Tema 2. Unidad 1. Presentación (V, ST, MA [T, D]).

- Presentarse informalmente (VS, MA [T, D, I]).

- Describir físicamente (VS, MA[T, D, I]).

- Actividad. Escucha y repite. Descripciones (V).

- Actividad. Ser o tener (ST, MA [I]).

- Actividad. Léxico (ST [I]).

- Actividad. Descripción (ST [I]).

- Actividad. Verbo ser(ST [I]).

- Actividad. Comprensión y expresión escrita (ST [I]).

- Final Tema 2. Unidad 1 (T, MA [R]).

- Actividad. Completa el diálogo (EP).

BT6. Gente que conocemos II. Dar información sobre amigos y familiares

- Tema 2. Unidad 2. Objetivos y competencias (T).

- Tema 2. Unidad 2. Presentación (V, ST, MA [T, D]).

- Describir el carácter (VS, MA [T, D, I]).

- Actividad. Escucha y marca. Adjetivos (V, ST).

- Actividad. Escucha y repite. Adjetivos (V).

- Actividad. Adjetivos (ST [I]).

- Hablar de la familia (VS, MA [T, D, I]).

- Actividad. Verdadero o falso (ST [I]).

- Actividad. Escucha y marca. Familia (V, ST).

- Actividad. Escucha y repite. Miembros de la familia (V).

- Actividad. Familia (ST [I]).

- Actividad. Escucha y repite. Adjetivos posesivos (V).

- Actividad. Gramática (ST [I]).

- Final Tema 2. Unidad 2 (T, MA [R]).

- Actividad: Completa el diálogo (EP).

BT7. Gente que conocemos III. Hacer presentaciones sobre gente que conocemos, pedir y dar información sobre el estado físico.

- Tema 2. Unidad 3. Objetivos y competencias (T).

- Tema 2. Unidad 3. Presentación (V, ST, MA [T, D]).

- Presentar a gente (VS, MA [T, D, I]).

- Hablar del estado físico (VS, MA [T, D, I]).

- Actividad. Escucha y repite. Estar o tener (V).

- Actividad. Completa con estar o tener (ST [I]).

- Final Tema 2. Unidad 3. (T, MA [R]).

- Actividad. Completa el diálogo (EP). 
BT8. Gente que conocemos IV. Hablar de gustos y actividades, expresar deseos.

- Tema 2. Unidad 4. Objetivos y competencias (T).

- Tema 2. Unidad 4. Presentación (V, ST, MA[T, D]).

- Preguntar por gustos y expresar gustos (VS, MA [T, D, I]).

- Actividad. Escucha y repite. Gustos y preferencias (V).

- Actividad. Completa con gusta o gustan (ST [I]).

- Actividad. Ordena las frases (ST [I]).

- Final Tema 2. Unidad 4 (T, MA [R]).

- Actividad. Completa el diálogo (EP).

- Tema 2. Unidad 5. Objetivos y competencias (T).

- Tema 2. Unidad 5. Presentación (V, ST, MA [T, D]).

- Presentar a alguien (VS, MA [T, D, I]).

- Describir a otras personas/Preguntar por otras personas (VS, MA [T, D, I]).

- Actividad. Descripción física (ST, MA [I]).

- Actividad. Completa con muy, mucho, mucha, muchos, muchas (ST [I]).

- Actividad. Elige la respuesta correcta (ST [I]).

- Final Tema 2. Unidad 5 (T, MA [R]).

- Actividad. Completa el diálogo (EP).

BT9. El entorno, ciudades y barrios I. Hablar y preguntar por una dirección, hablar del origen y del destino.

- Tema 3. Unidad 1. Objetivos y competencias (T).

- Tema 3. Unidad 1. Presentación (V, ST, MA [T, D]).

- Preguntar por una dirección I (VS, MA [T, D, I]).

- Preguntar por una dirección II (VS [T, D]).

- Preguntar por una dirección III (VS [T, D]).

- Preguntar por una dirección IV (VS, MA [T, D, I]).

- Preguntar por una dirección V (VS [T, D]).

- Preguntar por una dirección VI (VS, MA [T, D, I]).

- Preguntar por una dirección VII (VS, MA [T, D, I]).

- Actividad. Verbos estar, salir, ir, girar (ST [I]).

- Actividad. Direcciones (ST [I]).

- Actividad. Escucha y repite. Hablar y preguntar por una dirección (V).

- Actividad. Relaciona las direcciones (ST [I]).

- Actividad. Hay, está, están (ST [I]).

- Actividad. Correcto o incorrecto (ST [I]).

- Final Tema 3. Unidad 1 (T, MA [R]).

- Actividad. Completa el diálogo (EP). 
BT10. El entorno, ciudades y barrios II. Preguntar por una dirección, comprender instrucciones, indicar el camino y medios de transporte.

- Tema 3. Unidad 2. Objetivos y competencias (T).

- Tema 3. Unidad 2. Presentación (V, ST, MA [T, D]).

- Preguntar por una dirección y entender las instrucciones (VS, MA [T, D, I]).

- Indicar el camino (VS, MA [T, D, I]).

- Actividad. Completa con estar, bajar, coger, llegar, vivir o llamarse (ST [I]).

- Actividad. Escucha y repite. Hablar de medios de transporte (V).

- Actividad. Relaciona los medios de transporte (ST, MA [I]).

- Actividad. Completa con haber o tener (ST [I]).

- Actividad. Señala la opción correcta (ST [I])

- Actividad. Completa las frases con la palabra (ST [I]).

- Final Tema 3. Unidad 2 (T, MA [R]).

- Actividad: completa el diálogo (EP).

BT11. El entorno, ciudades y barrios III. Expresar distancia. Expresar frecuencia.

- Tema 3. Unidad 3. Objetivos y competencias (T).

- Tema 3. Unidad 3. Presentación (V, ST, MA [T, D]).

- Expresar distancia (VS, MA [T, D, I]).

- Expresar frecuencia (VS, MA [T, D, I]).

- Actividad. ¿Frecuencia o distancia? (ST [I]).

- Actividad. Comprensión oral (ST [T]).

- Actividad. Preposiciones (ST, MA [I]).

- Final Tema 3. Unidad 3. (T, MA [R]).

- Actividad. Completa el diálogo (EP).

BT12. El entorno, ciudades y barrios IV. Preguntar y pedir información.

- Tema 3. Unidad 4. Objetivos y competencias (T).

- Tema 3. Unidad 4. Presentación (V, ST, MA [T, D]).

- Pedir información (VS, MA [T, D, I]).

- Pedir información sobre una ciudad (VS, MA [T, D, I]).

- Pedir información concreta (VS, MA [T, D, I]).

- Actividad. Tipos de información (ST [I]).

- Actividad. Ordena las frases (ST [I]).

- Actividad. Escucha y repite (V).

- Final Tema 3. Unidad 4 (T, MA [R]).

- Actividad. Completa el diálogo (EP).

- Tema 3. Unidad 5. Objetivos y competencias (T). 
- Tema 3. Unidad 5. Presentación (V, ST, MA [T, D]).

- Describir una ciudad (VS, MA [T, D, I]).

- Actividad. Escucha y repite. Describir lugares (V).

- Actividad. Marca el verbo correcto (ST [I]).

- Actividad. Antónimos (ST [I]).

- Actividad. Completa con ser o tener (ST [I]).

- Final Tema 3. Unidad 5 (T, MA [R]).

- Actividad. Completa el diálogo (EP).

BT13. Actividad social: comidas y bebidas I. Hablar de comidas y bebidas, expresar preferencias.

- Tema 4. Unidad 1. Objetivos y competencias (T).

- Tema 4. Unidad 1. Presentación (V, ST, MA [T, D]).

- Pedir algo para tomar (VS, MA [T, D, I]).

- Hablar de gustos y preferencias (VS, MA [T, D, I]).

- Hablar de alimentos y sus ingredientes (VS, MA [T, D, I]).

- Actividad. Escucha y repite. Hablar de comida (V).

- Actividad. Marca y clasifica (ST [I]).

- Actividad. Escucha y repite. Gustos y preferencias (V).

- Actividad. Verbos preferir y querer (ST [I]).

- Actividad. Verbos gustar, preferir y querer (ST [I]).

- Final Tema 4. Unidad 1 (T, MA [R]).

- Actividad. Completa el diálogo (EP).

BT14. Actividad social: comidas y bebidas II. Preguntar por un plato o una tapa, hablar de cantidades.

- Tema 4. Unidad 2. Objetivos y competencias (T).

- Tema 4. Unidad 2. Presentación (V, ST, MA [T, D]).

- Hablar de cantidades (VS, MA [T, D, I]).

- Preguntar por un plato o tapa (VS, MA [T, D, I]).

- Actividad. Escucha y repite. Preguntar por un plato o describir un plato (V).

- Actividad. Relaciona pregunta-respuesta (ST [I]).

- Actividad. Verdadero o falso (ST [I]).

- Final Tema 4. Unidad 2 (T, MA [R]).

- Actividad. Completa el diálogo (EP).

BT15. Actividad social: comidas y bebidas III. Hablar de las horas y los momentos del día; comportamientos sociales.

- Tema 4. Unidad 3. Objetivos y competencias (T).

- Tema 4. Unidad 3. Presentación (V, ST, MA [T, D]).

- Hablar de horas y momentos del día (VS, MA [T, D, I]). 
- Comportamientos sociales (VS, MA [T, D, I]).

- Actividad. Escucha y repite. Expresar frecuencia (V).

- Actividad. Escucha y repite. Los números (V).

- Actividad. Escribe el número (ST [I]).

- Actividad. Escucha y repite. Horarios (V).

- Actividad. Relaciona las comidas con los momentos del día (ST [I]).

- Actividad. Preposiciones (ST [I]).

- Actividad. Días de la semana (ST [I]).

- Final Tema 4. Unidad 3 (T, MA [R]).

- Actividad. Completa el diálogo (EP).

BT16. Actividad social: comidas y bebidas IV. Pedir la cuenta, hablar de costumbres.

- Tema 4. Unidad 4. Objetivos y competencias (T).

- Tema 4. Unidad 4. Presentación (V, ST, MA [T, D]).

- Pedir la cuenta (VS, MA [T, D, I]).

- Hablar de la propina (VS, MA [T, D, I]).

- Actividad. Escucha y repite. Pedir la cuenta (V).

- Actividad. Ordena las palabras (ST [I]).

- Final Tema 4. Unidad 4 (T, MA [R]).

BT17. Hábitos para el tiempo libre I. Describir hábitos y costumbres en vacaciones.

- Tema 5. Unidad 1. Objetivos y competencias (T).

- Tema 5. Unidad 1. Presentación (V, ST, MA [T, D]).

- Pedir y dar información sobre la frecuencia con la que se realiza algo (VS, MA [T, D, I]).

- Describir hábitos y costumbres (VS, MA [T, D, I]).

- Actividad. Actividades para realizar en vacaciones (ST [I]).

- Actividad. Verbos reflexivos (ST [I]).

- Actividad. Pronombres (ST [I]).

- Final Tema 5. Unidad 1 (T, MA [R]).

- Actividad. Completa el diálogo (EP).

BT18. Hábitos para el tiempo libre II. Expresar gustos y preferencias, hacer una propuesta.

- Tema 5. Unidad 2. Objetivos y competencias (T).

- Tema 5. Unidad 2. Presentación (V, ST, MA [T, D]).

- Hacer una propuesta (VS, MA [T, D, I]).

- Expresar gustos y preferencias (VS, MA [T, D, I]).

- Actividad. Escucha y repite. Hacer una propuesta (V). 
- Actividad. Escucha y repite. Meses del año (V).

- Actividad. Los meses y estaciones del año.

- Actividad. Marca y clasifica.

- Final Tema 5. Unidad 2 (T, MA [R]).

- Actividad. Completa el diálogo (EP).

BT19. Hábitos para el tiempo libre III. Recomendar lugares, hablar del tiempo.

- Tema 5. Unidad 3. Objetivos y competencias (T).

- Tema 5. Unidad 3. Presentación (V, ST, MA[T, D]).

- Recomendar lugares (VS, MA [T, D, I]).

- Hablar del tiempo (VS, MA [T, D, I]).

- Proponer sitios y opinar (VS, MA [T, D, I]).

- Actividad. Escucha y repite. Tiempo atmosférico (V).

- Actividad. Ordena las frases (ST [I]).

- Cultura española (I).

- Final Tema 5. Unidad 3. (T, MA [R]).

BT20. Hábitos para el tiempo libre IV. Describir y comparar actividades.

- Tema 5. Unidad 4. Objetivos y competencias (T).

- Tema 5. Unidad 4. Presentación (V, ST, MA [T, D]).

- Comparar actividades (VS, MA [T, D, I]).

- Actividad. Escucha y repite. Comparar actividades (V).

- Actividad. Léxico (ST [I]).

- Final Tema 5. Unidad 4 (T, MA [R]).

- Actividad. Completa el diálogo (EP).

- Tema 5. Unidad 5. Objetivos y competencias (T).

- Tema 5. Unidad 5. Presentación (V, ST, MA [T, D]).

- Hablar de actividades en grupo (VS, MA [T, D, I]).

- Hacer valoraciones (VS, MA [T, D, I]).

- Actividad. Escucha y repite. Hacer valoraciones (V).

- Actividad. Relaciona (ST [I]).

- Actividad. Correcto o incorrecto (ST [I]).

- Final Tema 5. Unidad 5 (T, MA [R]).

EF. 


\section{Encuesta}

The following survey is intended to know your opinion and insights of the MOOC Spanish for Beginners launched in http://iversity.org. The survey is composed by 10 questions. Each question uses Likert scale from 1 (totally disagreed) to 5 (totally agreed). Please, answer the next 10 questions about our MOOC. We kindly appreciate your answers in order to improve the next edition Spanish for Beginners. Your opinion is really important for us. Thank you!

Q1. Did you have fun with this course?*

$\begin{array}{lcccccc} & 1 & 2 & 3 & 4 & 5 & \\ \text { Totally disagreed } & \bigcirc & 0 & \bigcirc & \bigcirc & \bigcirc & \text { Totally agreed }\end{array}$

\section{Q2. Are the activities (quizzes and homework) correctly explained?*}

$\begin{array}{lllllll} & 1 & 2 & 3 & 4 & 5 & \\ \text { Totally disagreed } & 0 & 0 & 0 & \bigcirc & 0 & \text { Totally agreed }\end{array}$

Q3. Do you think the contents of this course are appropriate to learn Spanish?*

$\begin{array}{lcccccc} & 1 & 2 & 3 & 4 & 5 & \\ \text { Totally disagreed } & \bigcirc & 0 & \bigcirc & \bigcirc & \bigcirc & \text { Totally agreed }\end{array}$

Q4. Do you think there are many activities (quizzes and homework) in this course?*

$\begin{array}{lcccccc} & 1 & 2 & 3 & 4 & 5 & \\ \text { Totally disagreed } & - & 0 & 0 & \bigcirc & \bigcirc & \text { Totally agreed }\end{array}$

Q5. With regards to homework activities, do you think the number of these activities is appropriate?*



Q6. Did you have problems with homework activities?*

$\begin{array}{lcccccc} & 1 & 2 & 3 & 4 & 5 & \\ \text { Totally disagreed } & & 0 & \bigcirc & \bigcirc & \bigcirc & \text { Totally agreed }\end{array}$


$\ldots / \ldots$

Q7. Did you have problems with quizzes?*

$\begin{array}{ccccccc} & 1 & 2 & 3 & 4 & 5 \\ \text { Totally disagreed } & 0 & 0 & 0 & 0 & \bigcirc & \text { Totally agreed }\end{array}$

Q8. Did you like the videos?*

$\begin{array}{lllllll}1 & 2 & 3 & 4 & 5 \\ \text { Totally disagreed } & 0 & 0 & 0 & 0 & 0 & \text { Totally agreed }\end{array}$

Q9. Do you think the subtitles in videos did help you to understand the contents of this course?*

$\begin{array}{ccccccc}1 & 2 & 3 & 4 & 5 \\ \text { Totally disagreed } & 0 & 0 & 0 & 0 & 0 & \text { Totally agreed }\end{array}$

Q10. The course was designed in Spanish language with the aim that students are being integrated in the Spanish environment. However, the announcements and discussions were in English language. Do you think the English language could help you to improve your performance in this course?*

$\begin{array}{ccccccc}1 & 2 & 3 & 4 & 5 \\ \text { Totally disagreed } & 0 & 0 & 0 & 0 & \bigcirc & \text { Totally agreed }\end{array}$

* Required. 


\section{Relación de ciclos formativos de grado superior para cursar grados en la UDIMA}

La Universidad a Distancia de Madrid (UDIMA) tiene aprobado el reconocimiento de los ciclos formativos de grado superior para cursar las carreras universitarias indicadas por la Consejería de Educación de la Comunidad de Madrid:

\section{Para el Grado en Ingeniería de Organización industrial}

- Administración y Finanzas.

- Administración de Sistemas Informáticos.

- Automatización y Robótica Industrial.

- Automoción.

- Construcciones Metálicas.

- Desarrollo de Productos Electrónicos.

- Desarrollo de Proyectos Mecánicos.

- Diseño en Fabricación Mecánica.

- Instalaciones Electrotécnicas.

- Mantenimiento Aeromecánico.

- Mantenimiento de Equipo Industrial.

- Mantenimiento y Montaje de Instalaciones de Edificio y Proceso.

- Mantenimiento de Instalaciones Térmicas y de Fluidos.

- Mecatrónica Industrial.

- Industrias de Proceso Químico.

- Producción por Mecanizado.

- Programación de la Producción en Fabricación Mecánica.

- Química Industrial.

- Sistemas Electrotécnicos y Automatizados.

- Sistemas de Regulación y Control Automáticos.

- Sistemas de Telecomunicaciones e Informáticos.
Para el Grado en Ingeniería de Tecnologías y Servicios de Telecomunicación

- Administración de Sistemas Informáticos.

- Automatización y Robótica Industrial.

- Desarrollo de Aplicaciones Informáticas.

- Desarrollo de Aplicaciones Multiplataforma.

- Desarrollo de Aplicaciones Web.

- Desarrollo de Productos Electrónicos.

- Instalaciones Electrotécnicas.

- Mantenimiento Electrónico.

- Sistemas Electrotécnicos y Automatizados.

- Sistemas de Regulación y Control Automáticos.

- Sistemas de Telecomunicaciones e Informáticos

Para los Grados en Derecho o en Ciencias del Trabajo, Relaciones Laborales y Recursos Humanos

- Administración y Finanzas.

- Asistencia a la Dirección.

- Secretariado. 


\section{para cursar grados en la UDIMA}

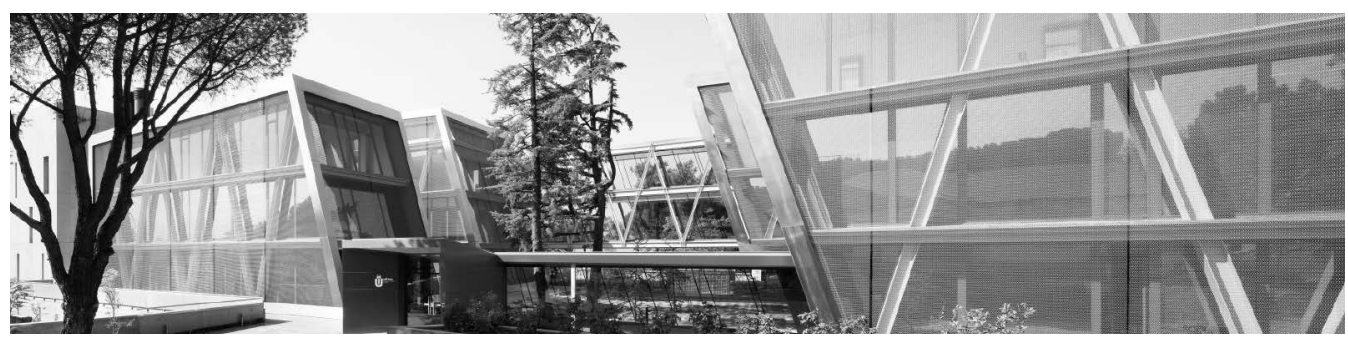

\section{< > Para el Grado en Ingeniería Informática}

- Administración de Sistemas Informáticos.

- Automatización y Robótica Industrial.

- Desarrollo de Aplicaciones Informáticas.

- Desarrollo de Aplicaciones Multiplataforma.

- Desarrollo de Aplicaciones Web.

- Sistemas de Telecomunicaciones e Informáticos.

\section{Para los Grados en Administración y Dirección de Empresas o en Economía}

- Administración y Finanzas.

- Asistencia a la Dirección.

- Comercio Internacional.

- Gestión Comercial y Marketing.

- Marketing y Publicidad.

- Secretariado.

- Transporte y Logística.

\section{Para el Grado en Marketing}

- Administración y Finanzas.

- Comercio Internacional.
- Gestión Comercial y Marketing.

- Gestión de Alojamientos Turísticos.

- Gestión de Ventas y Espacios Comerciales.

- Marketing y Publicidad.

- Transporte y Logística.

Para los Grados en Magisterio de Educación Infantil y de Educación Primaria

- Animación de Actividades Físicas y Deportivas.

- Educación Infantil.

- Integración Social.

- Animación Sociocultural y Turística.

\section{Para el Grado en Empresas y} Actividades Turísticas

- Gestión Comercial y Marketing.

- Gestión de Ventas y Espacios Comerciales.

- Gestión de Alojamientos Turísticos.

- Agencias de Viajes y Gestión de Eventos.

- Guía, Información y Asistencias Turísticas. 